\title{
Spectroscopic orbits of nearby stars ${ }^{\star}$
}

\author{
J. Sperauskas ${ }^{1}$, V. Deveikis ${ }^{1}$, and A. Tokovinin ${ }^{2}$ \\ 1 Vilnius University Observatory, Saulètekio al. 3, 10257 Vilnius, Lithuania \\ e-mail: julius.sperauskas@ff.vu.lt \\ 2 Cerro Tololo Inter-American Observatory, Casilla 603, La Serena, Chile \\ e-mail: atokovinin@ctio.noao.edu
}

Received 22 February 2019 / Accepted 22 March 2019

\begin{abstract}
Aims. We observed stars with variable radial velocities to determine their spectroscopic orbits.

Methods. Velocities are presented of 132 targets taken over a time span reaching 30 years. These were measured with the correlation radial velocity spectrometers (1917 velocities) and the new VUES echelle spectrograph (627 velocities), with a typical accuracy of 0.5 and $0.2 \mathrm{~km} \mathrm{~s}^{-1}$, respectively.

Results. We derived spectroscopic orbits of 57 stars (including 53 first-time orbits), mostly nearby dwarfs of spectral types $\mathrm{K}$ and $\mathrm{M}$, with some being HipPaRcos astrometric binaries. Their periods range from 2.2 days to 14 years. Comments on individual objects are provided. Many stars belong to hierarchical systems containing three or more components, including 20 new hierarchies resulting from this project. The preliminary orbit of the young star HIP $47110 \mathrm{~B}$ has a large eccentricity $e=0.47$ despite having a short period of $4.4 \mathrm{~d}$; it could still be circularizing.

Conclusions. Our results enrich the data on nearby stars and contribute to a better definition of the multiplicity statistics.
\end{abstract}

Key words. binaries: close

\section{Introduction}

The solar neighborhood is the best-studied part of the Galaxy. Yet, it is still a site of active research and new discoveries. While the Gaia satellite is improving the census of our stellar neighbors, several observational campaigns target nearby stars in search of exo-planets. Studies of specific stellar populations, such as young associations or metal-poor stars, inevitably focus on the nearest objects. Last but not least, the solar neighborhood is the benchmark for stellar multiplicity statistics.

Here we report the results of the large campaign of radial velocity (RV) measurements, which started three decades ago, before the HIPPARcos mission, targeting mostly nearby low-mass stars. The aims and main results of this campaign are presented by Sperauskas et al. (2016), where the stars with constant RVs are featured. Here we focus on the remaining objects with variable (or supposedly variable) RVs. Our goal is the determination of spectroscopic orbits of these stars.

Radial velocity monitoring over several years is very efficient in discovering spectroscopic binaries (SBs); only a few (e.g., three) RV measurements are needed to detect the RV variability or double lines. However, determination of orbits of these SBs requires substantial follow-up efforts. For example, the Geneva-Copenhagen Survey (GCS; Nordström et al. 2004) discovered hundreds of SBs, but their orbits remain for the most part unknown or unpublished. This leads to uncertainties in the study of stellar multiplicity, namely in the distribution of periods and mass ratios of nearby solar-type stars (Tokovinin 2014).

Nowadays, hundreds of thousands of RV measurements come from Gaia (Gaia Collaboration 2018) and ground-based

\footnotetext{
* Full Table 1 is only available at the CDS via anonymous $\mathrm{ftp}$ to cdsarc.u-strasbg. fr (130.79.128.5) or via http://cdsarc. u-strasbg.fr/viz-bin/qcat?]/A+A/626/A31.
}

surveys such as APOGEE (e.g., Albareti et al. 2017), RAVE (Steinmetz et al. 2006), or LAMOST (Zhao et al. 2012). However, some SB orbits require either a long time span or a frequent cadence, which are not provided by the automatic surveys. So far, only Gaia offers the full-sky coverage, but it has not yet provided individual RV measurements suitable for orbit calculation. Moreover, treatment of double-lined systems and complex cases such as triples by the Gaia pipeline may be problematic. Our results are therefore unlikely to become obsolete in the near term.

The results of our observations are useful in many ways. They provide previously unknown periods and mass ratios of lowmass binaries in the solar neighborhood that serve to improve the multiplicity statistics. The recent discovery of the strong dependence of the close-binary fraction on metallicity (Moe et al. 2019) puts such efforts in a new context. Several binaries detected by Hipparcos accelerations have their spectroscopic orbits determined here. Similarly, this dataset will help in the interpretation of astrometric accelerations measured by Gaia, as our time coverage is much longer than the duration of this mission. Finally, some objects in our sample present special interest for various reasons, making the knowledge of their orbits essential.

The objects of this study are presented in Sect. 2. Section 3 covers the instruments and methods used to derive the orbits. Our results, namely the orbits and comments on individual objects, are given in Sect. 4. Finally, our conclusions are presented in Sect. 5.

\section{The observed sample}

The core of the observing program is the survey of $\mathrm{K}$ - and M-type dwarfs featured in the McCormick catalog (see a review in Upgren et al. 1989) and in the catalog of nearby stars 


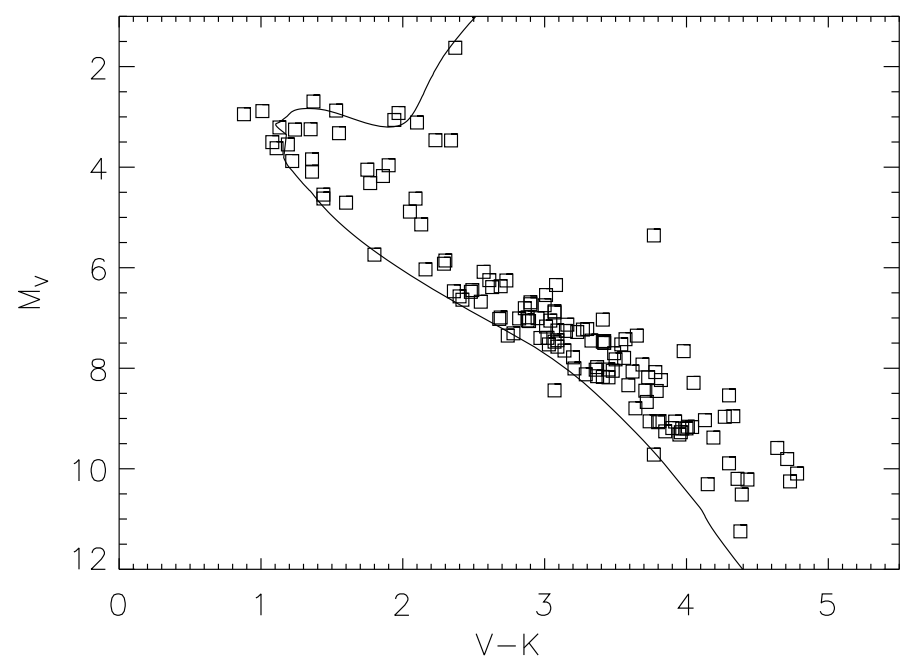

Fig. 1. Color-magnitude diagram of stars from Table A.1. The line is a 4 Gyr isochrone for solar metallicity from Bressan et al. (2012). The discrepant point is caused by the erroneous parallax of BD-08 2689.

(Gliese \& Jahreiß 1991). Stars with constant RVs presented by Sperauskas et al. (2016) were used to study the local kinematics. Objects with variable RVs studied here were monitored more extensively for orbit determination. The spectroscopic survey of nearby K dwarfs by Halbwachs et al. $(2003,2018)$ pursued similar goals, and six of their objects are common to our sample. In addition to the nearby dwarfs, we monitored several other objects with variable RVs and present their orbits here. In particular, our program was augmented by the HIPPARCos stars with astrometric accelerations (Makarov \& Kaplan 2005) with the aim being to establish their periods.

Table A.1 contains the object list and the synopsis of our results. Its first column gives common identifiers (HIP numbers are preferred, with HD or BD as the second choice), and the following two columns give the equatorial coordinates for J2000. Then follow the visual magnitude $V$, parallax $\varpi$, and spectral type. These data were recovered from Simbad. Almost all parallaxes come from the Gaia DR2 (Gaia Collaboration 2018). The remaining columns of Table A.1 summarize our results. The variability is coded as $\mathrm{C}$ - constant, $\mathrm{V}$ - variable, $\mathrm{s} 2$ - doublelined, S1, S2, or S3 - single-, double-, and triple-lined binaries with orbits determined here. Then follow the number of RV measurements $N$, their time span $\Delta T$, and the weighted mean velocity $\langle R V\rangle$. For binaries with orbits, $\langle R V\rangle$ is the center-of-mass velocity. The last two columns contain the statistics explained below.

To give an idea of the stars studied here, we place them on the color-magnitude diagram in Fig. 1. Most stars are low-mass dwarfs, although hotter F-type stars and giants are also present in our sample. We reiterate that at the start of our program, the trigonometric parallaxes were not available, and some stars classified spectroscopically as dwarfs turned out to be giants. The median parallax is 25 mas, meaning that half of the objects are located within $40 \mathrm{pc}$ of the Sun. The closest, HIP 29295, has a distance of only $5.7 \mathrm{pc}$. On the other hand, 16 objects (mostly giants) have parallaxes less than 5 mas.

\section{Observations and data processing}

\subsection{Instruments}

The first RV measurements reported here date back to February 1988. They were made using the correlation radial-velocity

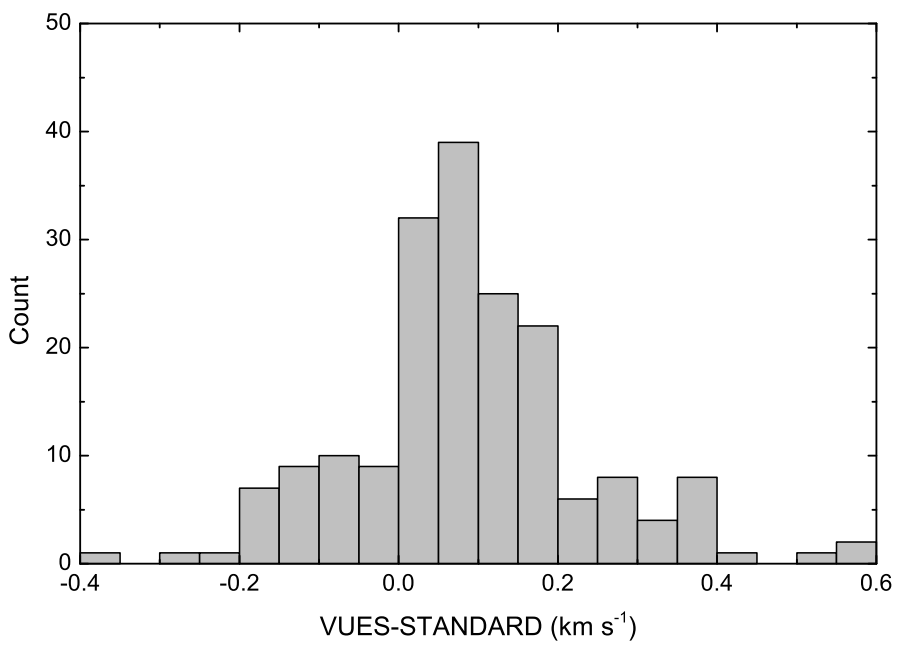

Fig. 2. Radial velocities of IAU RV standard stars measures by VUES. The histogram of the RV difference is plotted.

meter (RVM) installed at the $1 \mathrm{~m}$ Lithuanian telescope at Mt. Maidanak, in Uzbekistan (see e.g., Tokovinin 1992). Like the CORAVEL instrument (Baranne et al. 1979), it scans the echelle spectrum over the physical mask with slits corresponding to individual spectral lines, accumulates the transmitted flux as a function of the relative shift, and determines the RV by approximating the cross-correlation curve with one or several Gaussian curves.

Starting from 1998, a similar CORAVEL-type instrument constructed at the Vilnius observatory became operational. It worked mostly at the $1.65 \mathrm{~m}$ telescope of the Moletai observatory, although several trips to other telescopes were made. The instrument and observing runs are further described by Sperauskas et al. (2016). This latter publication also gives a thorough analysis of the RV zero points and accuracy by comparing results to several lists of RV standards. In the following, we refer to both instruments as CORAVELs, without making distinction between them.

In 2015, the CORAVEL in Moletai was replaced by the modern fiber-fed echelle spectrometer VUES (Jurgenson et al. 2016). It covers the spectral range from 400 to $880 \mathrm{~nm}$ with a resolution from 30000 to 60000 . In this program we used the lowest resolution of 30000 . The first RV measurement with VUES reported here was made on November 26, 2015 (JD 2457352). The spectrum recorded by the CCD detector is extracted and calibrated in the standard way. The RV is determined by numerical cross-correlation of this spectrum with a binary mask, emulating the CORAVEL method in software. Compared to CORAVEL, the RVs delivered by VUES are more accurate; their rms residuals from the orbits are typically from 0.2 to $0.3 \mathrm{~km} \mathrm{~s}^{-1}$. The RV zero point is controlled by observations of the IAU RV standards (Fig. 2). The systematic RV offset of VUES is less than $0.1 \mathrm{~km} \mathrm{~s}^{-1}$.

\subsection{Detection of variable RVs}

For each star, the mean radial velocity $\langle R V\rangle$ was computed with weights inversely proportional to the squares of the measurement errors. The errors of the CORAVEL RVs $\sigma_{i}$ were used as listed, while the errors of the RVs measured by VUES were augmented by adding quadratically $0.2 \mathrm{~km} \mathrm{~s}^{-1}$ because the internal errors determined by the dip fitting do not account for other error sources such as wavelength calibration and instrument stability. 
Table 1. Radial velocities.

\begin{tabular}{lccccc}
\hline \hline Name & $\begin{array}{c}\text { JD } \\
+2400000\end{array}$ & $\begin{array}{c}\text { RV } \\
\left(\mathrm{km} \mathrm{s}^{-1}\right)\end{array}$ & $\begin{array}{c}\sigma \\
\left(\mathrm{km} \mathrm{s}^{-1}\right)\end{array}$ & Inst. & $\mathrm{a} / \mathrm{b}$ \\
\hline HIP 96 & 55470.486 & -11.70 & 0.60 & $\mathrm{C}$ & \\
HIP 96 & 55485.410 & -11.60 & 0.50 & $\mathrm{C}$ & \\
HIP 3428 & 58387.512 & -1.09 & 0.42 & $\mathrm{~V}$ & $\mathrm{a}$ \\
HIP 3428 & 58387.512 & -14.29 & 0.53 & $\mathrm{~V}$ & $\mathrm{~b}$ \\
\hline
\end{tabular}

Notes. The full table is available at the CDS.

The weighted rms deviation from the mean, $\sigma_{V}$, is computed as

$\sigma_{v}=\sum_{i=1}^{N}\left(R V_{i}-\langle R V\rangle\right)^{2} \sigma_{i}^{-2} / \Sigma_{i=1}^{N} \sigma_{i}^{-2}$.

The first term of this equation, divided by the number of measurements $N-1$, gives the normalized $\chi^{2} /(N-1)$ statistic, which has a mathematical expectation of one for a constant RV and realistic errors $\sigma_{i}$. The statistics $\sigma_{V}$ and $\chi^{2} /(N-1)$ are given in the last two columns of Table A.1, except for the stars with computed orbits. The large values $\chi^{2} /(N-1)>100$ are replaced by 99. For multi-lined systems, the statistics are computed for the primary component.

\subsection{Orbit calculation}

Spectroscopic orbits were determined with the help of the IDL code orbit.pro ${ }^{1}$. Individual RVs are weighted in proportion to $\sigma_{i}^{-2}$. However, the errors are artificially increased when RVs are deduced from partially blended dips and in other instances where the residuals strongly exceed the errors. In a few cases where the spectroscopic pair is also resolved, we fitted combined spectro-visual orbits using the same code. The errors of $\mathrm{RVs}$ and positional measurements are balanced in the sense that the normalized statistic $\chi^{2} / N$ for each type of data should be close to one. Finally, orbits of triple systems were fitted using an extension of this code called orbit3.pro and described by Tokovinin \& Latham (2017); this is also available online.

\section{Results}

\subsection{Individual RVs}

Table 1, published in full at the CDS, lists individual RV measurements, a total of 1917 velocities obtained with CORAVEL, and 627 velocities measured with VUES. Its first column is the object name (same as in the object list). Then follow the Julian date, RV, its internal error, and the instrument code (V for VUES and $\mathrm{C}$ for CORAVEL). For double-lined binaries, the last column distinguishes the primary and secondary components by the letters a and $b$, respectively.

\subsection{Spectroscopic orbits}

The orbital elements and their errors are listed in Table A.2 in standard notation. For circular orbits, we fixed the eccentricity $e$ and the argument of periastron $\omega$. The second-to-last column gives the weighted rms residuals for the primary component or

\footnotetext{
1 The code can be downloaded from http://www. ctio.noao.edu/ atokovin/orbit/
}

for both components of double-lined (SB2) binaries. The spectroscopic masses $M_{1,2} \sin ^{3} i$, that is, the minimum masses, are provided for SB2s in the last column. For the single-lined pairs (SB1s), this column contains the minimum secondary mass estimated from the orbit after adopting a reasonable guess for the primary mass.

The RV curves of spectroscopic binaries are given in Fig. A.1. In each panel, the horizontal axis is the orbital phase from 0 to 1.5 (the first half-period is repeated), the vertical axis is the RV in $\mathrm{km} \mathrm{s}^{-1}$. The RV curves of the primary and secondary components are plotted in full and dashed lines, respectively, while the squares and triangles denote the measurements. In some plots, crosses denote RVs with reduced weights.

\subsection{Comments on the individual objects}

This section provides notes on individual stars and stellar systems from our list. Hierarchical systems with three or more components are also featured in the Multiple-Star Catalog (MSC; Tokovinin 2018). Several objects with subsystems discovered here are added to the MSC. Data on visual components are taken from the Washington Double Star catalog (WDS; Mason et al. 2001) and from the MSC ${ }^{2}$. Unknown periods of visual binaries are estimated crudely from projected separations assuming that they equal the semimajor axis. Similary, the semimajor axes of spectroscopic binaries are estimated from their periods, using known distance and a guess of the masses of the components. Information on astrometric accelerations detected by the HipPARcos mission comes from the paper by Makarov \& Kaplan (2005).

HIP 96 (BD+13 5195, M0.5V, 43 pc). This visual triple system consists of the $11^{\prime \prime}$ pair A,B and the 0 '.2 subsystem Aa,Ab with $P \sim 25$ yr. All components are M-type dwarfs. We find that the RVs of both A and B are slowly variable. This is expected for A, which is a close pair; the component B may also host a low-mass companion.

HIP 374 (HD 225220), a K0 giant, is the main component A of the hierarchical system located at a distance of $200 \mathrm{pc}$. The outer pair A,D has a 95'!3 separation; A,B is a visual binary with $P=545 \mathrm{yr}$. The main sequence star D (TYC 2267-1300-1, probably of F9V spectral type) is found here to be an SB1 with $P=68 \mathrm{~d}$. This is therefore a quadruple system of $2+2$ hierarchy. The star C = HIP 375, listed in the WDS, is optical, as evidenced by its proper motion (PM), different parallax, and the RV measured here.

HIP $1412(\mathrm{~K} 7 \mathrm{~V}, 32 \mathrm{pc})$ has a large RV variation, but no orbit can be derived yet from our six RVs.

HIP $3428(\mathrm{BD}+2397, \mathrm{~K} 7,45 \mathrm{pc})$ is a double-lined twin binary with $P=97 \mathrm{~d}$ and the mass ratio $q=0.96$. Interestingly, it has an astrometric acceleration, and therefore is likely a triple system. However, residuals to the spectroscopic orbit do not show any slow trends.

HIP 5110 (HD 6440, 27 pc) is a 6".1 physical binary STF 87 with an estimated period of $\sim 2 \mathrm{kyr}$. We discover RV variability of the component B, of K8V spectral type. Considering also the $2.5 \mathrm{~km} \mathrm{~s}^{-1} \mathrm{RV}$ difference between $\mathrm{A}$ and $\mathrm{B}$, presumably caused by the orbital motion of $\mathrm{Ba}, \mathrm{Bb}$, we believe that this is a triple system.

$H D 8691$ (G0, $50 \mathrm{pc})$ is a high-PM star and an SB1 with $P=$ $581 \mathrm{~d}$, with a low-mass secondary.

\footnotetext{
2 See the latest version at http://www.ctio.noao.edu/
} atokovin/stars 
HIP 9867 (GJ 84.2, M0V, 19 pc) is a high-PM star and a double-lined pair with $P=897 \mathrm{~d}$ and unequal correlation dips. The WDS lists three visual companions, all optical. Eclipses are reported by Malkov et al. (2006).

HIP $10258(\mathrm{BD}+03301, \mathrm{~K} 5,47 \mathrm{pc})$ is a chromospherically active double-lined binary with $P=5.9 \mathrm{~d}$ and a mass ratio $q=$ 0.93. Its visual companion at 25" (SKF 1518) shares common parallax, PM, and RV.

$B D+49646$ (unknown spectral type, $53 \mathrm{pc}$ ) has double correlation dips, but we have not derived its orbit from the eight spectra. It is an X-ray source.

HIP 11437 (AG Tri, K7V, $41 \mathrm{pc}$ ) is a young chromospherically active star in the $\beta$ Pictoris moving group (Messina et al. 2017); it has been extensively covered in the literature. We found a constant RV of $6.0 \mathrm{~km} \mathrm{~s}^{-1}$ (see also Sperauskas et al. 2016), in agreement with other published studies. The visual companion at $22^{\prime \prime}$ is physical.

HIP 12787 (MCC 401, M0Ve, 49 pc) is a triple system with the outer $21^{\prime \prime}$ physical binary A,C. Its primary component is an astrometric binary, resolved directly in 2015.9 at $0^{\prime \prime} .25$ by Janson et al. (2017); its estimated period is $\sim 40 \mathrm{yr}$. The spectrum is double-lined, suggesting existence of a close subsystem, but no orbit is derived. The components $\mathrm{A}$ and $\mathrm{C}$ are located above the main sequence and belong to the $\beta$ Pictoris moving group according to Janson et al. The component B at $25^{\prime \prime}$ listed in the WDS is optical.

HIP 13398 (G 36-38, M2V, 23 pc) is a high-PM star, likely with a variable $\mathrm{RV}$.

HIP 13460 (BD+60 585, K3V, $39 \mathrm{pc}$ ) is a triple system discussed in the following section.

HIP 14478 (V568 Per, K6, 27 pc) is a triple system. The outer 2".9 pair A 1572 has an estimated period of $\sim 600 \mathrm{yr}$. Its primary component is a single-lined binary with $P=1325$ days. The semimajor axis of the inner subsystem is 87 mas and it is detectable astrometrically from the PM difference between Gaia and HiPparcos.

HIP 14669 (MCC 99, M2V, 17 pc) is a HiPPARCos visual binary with known orbit, $P=28.3 \mathrm{yr}$. We see an RV trend by $6 \mathrm{~km} \mathrm{~s}^{-1}$ over 7 years, presumably caused by this orbit.

HIP 14864 (BD+60 637, M0Ve, $25 \mathrm{pc})$ is a triple system consisting of the 0".6 binary discovered by HIPPARcos (period $\sim 40 \mathrm{yr}$ ) and the double-lined subsystem Aa,Ab with $P=59.5 \mathrm{~d}$ and $q=0.88$, discovered here.

$B D+03480$ (V1221 Tau, G0, $83 \mathrm{pc}$ ) is a young visual triple system, where the inner 0"9 binary A $2417 \mathrm{BC}$ has been known fora long time (since 1912), while another companion D at 2 '.2 was discovered a century later, in 2012, and has not yet been confirmed as physical (it is not found in Gaia DR2). Our 11 observations over 13 years show a constant RV of $12.66 \mathrm{~km} \mathrm{~s}^{-1}$. However, Gaia DR2 gives an RV of $18.05 \mathrm{~km} \mathrm{~s}^{-1}$ with an error of $7.26 \mathrm{~km} \mathrm{~s}^{-1}$, suggesting variability.

HIP 17102 (HD 278874, $39 \mathrm{pc}$ ) is a flaring K2V dwarf in a triple system. The outer pair ES 327 has a 15'.5 separation and a long $\sim 10 \mathrm{kyr}$ period. The secondary star B is located above the main sequence. The main component $\mathrm{A}$ is a double-lined binary with $P=183 \mathrm{~d}$ and $q=0.96$ (the components Aa and $\mathrm{Ab}$ are interchanged in our orbit). The inner semimajor axis is 18 mas, so the subsystem Aa,Ab can be resolved. Double lines were noted by Montes et al. (2018).

GJ 3248 is an M1V dwarf at 16 pc. We suspect that its RV is variable.

HIP $18448(\mathrm{~K} 0,149 \mathrm{pc})$ is a triple system composed of the outer $25^{\prime \prime}$ pair LDS 1583 and the inner subsystem Aa,Ab revealed by astrometric acceleration. Here we derive its accurate spectro- scopic orbit with $P=4.2 \mathrm{yr}$. The primary star is a subgiant located above the main sequence, while the component B is below; Chanamé \& Ramirez (2012) consider B to be a white dwarf.

HIP 19140 (BD-15 728, K5V, 40 pc) certainly has a variable $\mathrm{RV}$, as well as astrometric acceleration.

HIP 19915 (HD 26872, F8, 166 pc) is a triple system composed of the tight 32 mas interferometric pair YSC 128 with an estimated period of $\sim 6 \mathrm{yr}$ (no orbit is known yet) and the doublelined subsystem with $P=7.3$ days. The inner orbit is seen at large inclination, as evidenced by the small spectroscopic masses $M \sin ^{3} i$. We could not detect variations of the systemic velocity that might be caused by the visual binary.

$H D 279846$ (K2, $82 \mathrm{pc}$ ) is just a double-lined binary with $P=15.5$ days and an accurately determined orbit; $q=0.95$.

HIP 20709 (HD 27961, F5, 132 pc) is an interesting hierarchical system where both the outer $82 \mathrm{yr}$ visual orbit and the inner 141 day double-lined orbit, determined here, are known. The spectrum is triple-lined. We fit both orbits simultaneously, accounting for the slow RV drift caused by the visual pair HU 609. The inclination of the spectroscopic pair derived from comparison between the spectroscopic mass $M \sin ^{3} i$ and the mass estimated from absolute magnitude, is $60^{\circ}$ or $120^{\circ}$, while the outer inclination is $122^{\circ}$. The two orbits could therefore be coplanar. The inner semimajor axis is 5 mas, and therefore the system can be resolved with long-baseline interferometers like the CHARA array to measure the relative inclination. The rms scatter of RVs of the visual secondary component, B, is large: $2.3 \mathrm{~km} \mathrm{~s}^{-1}$. A possible orbit of $\mathrm{Ba}, \mathrm{Bb}$ with a $1600 \mathrm{~d}$ period and an amplitude of $2.3 \mathrm{~km} \mathrm{~s}^{-1}$ can be fitted, reducing the weighted rms to $0.6 \mathrm{~km} \mathrm{~s}^{-1}$. This tentative orbit is not given here. The minimum mass of the hypothetical component $\mathrm{Bb}$ is $0.15 \mathcal{M}_{\odot}$.

HIP 21710 (HD 286955, K2, $27 \mathrm{pc}$ ) is a nearby threetier quadruple system. The outer $34^{\prime \prime}$ pair A,B (GIC 51) has an estimated period of $23 \mathrm{kyr}$, the intermediate visual binary $\mathrm{Aa}, \mathrm{Ab}$ resolved by HipParcos has an orbit with $P=204 \mathrm{yr}$, and the inner spectroscopic binary has a period of 610 days announced by Halbwachs et al. (2003) and eventually published by Halbwachs et al. (2018). We determined the spectroscopic orbit from our own observations, but publish here more accurate elements derived from the combined data.

HIP 21845 (HD 29696, F8, 115 pc) might have a slow RV variation, although comparison between Gaia and HiPPARcos does not reveal any astrometric acceleration. The visual companion at $29^{\prime \prime}$ listed in the WDS is physical, according to its Gaia parallax and the RV of $11.4 \mathrm{~km} \mathrm{~s}^{-1}$ (the first discordant measure of this pair given in the WDS is misleading).

HIP 23550 (HD 32387, G8V, 73 pc) shows an RV trend over 9 years, indicative of a long period; it is an acceleration binary. The RV variability with an rms of $1.8 \mathrm{~km} \mathrm{~s}^{-1}$ was also detected by Niedever et al. (2002).

HIP 24488 (HD 33798, V390 Aur) is a visual binary with a period of $513 \mathrm{yr}$ according to its current (still uncertain) orbit. The Gaia parallax of 2.14 mas is erroneous, so the HipPARcos parallax of 8.73 mas is adopted, in better agreement with the dynamical parallax from the orbit, 6.0 mas. Fekel \& Marschall (1991) studied this lithium-rich, chromospherically active G5III giant and concluded that it is not a spectroscopic binary; they quote an RV of $22.5 \pm 0.2 \mathrm{~km} \mathrm{~s}^{-1}$. We noted doubling of some correlation dips that could be caused by the fast rotation, $V \sin i=$ $29 \mathrm{~km} \mathrm{~s}^{-1}$. The RVs derived from double dips are ignored here, and the RV is likely constant.

GJ 220 is a nearby M2V dwarf (parallax 51.5 \pm 4.6 mas) with a variable RV. We derived a tentative orbit with $P=700 \mathrm{~d}$ and 
$K_{1}=2 \mathrm{~km} \mathrm{~s}^{-1}$ from the 13 measured RVs. More observations are needed however to constrain our orbit before it can be published. Meanwhile, an orbit with $P=721.4 \mathrm{~d}$ was published by Baroch et al. (2018). The pair may have been resolved by Gaia because DR2 does not provide its parallax.

HIP 28663 (HD 41028, F4IV, 103 pc) is a single-lined binary with a well-determined orbit of $P=8.55 \mathrm{~d}$ and the minimum secondary mass of $0.35 \mathcal{M}_{\odot}$.

HIP 29295 (HD 42581, GJ 229) is a flaring M1V dwarf located at a distance of $5.8 \mathrm{pc}$. Its $\mathrm{RV}$ is constant over the 15.8 years spanned by our observations. There is extensive literature coverage of the search for exo-planets with precise RVs and photometry and the distant brown dwarf companion GJ 229B detected by imaging.

HIP $29316(\mathrm{BD}+101032)$ is an M3V nearby (11 pc) close visual binary KAM 1 with an estimated period of $\sim 20$ yr. Gaia DR2 does not provide astrometry for this resolved source. The $\mathrm{RV}$ is variable, possibly because of the visual orbit. The WDS companion $\mathrm{C}$ at $13^{\prime \prime}$ is optical.

HIP 30269 (HD 44517, F5V, 311 pc) has a large-amplitude RV variation discovered by Nordström et al. (2004), but a yet unknown orbit.

HIP 33560 (HD 51849, K4V, $22.5 \mathrm{pc}$ ) is a visual triple system composed of the $50^{\prime \prime}$ outer pair $A B, C$ and the $0 \prime \prime 6$ inner pair A,B discovered by HIPPARcos with an estimated period of $\sim 50 \mathrm{yr}$ but a yet unknown orbit. We suspect RV variability that might be caused by motion in the visual pair. The RV trend is also detected by Halbwachs et al. (2018). The star is featured in Sperauskas et al. (2016).

HIP 34341 (BD+03 1552, K5V, $26 \mathrm{pc})$ is a single-lined binary with $P=875 \mathrm{~d}$, as well as an astrometric binary.

HIP 35706 (BD+68 474, K5V, $42 \mathrm{pc}$ ) has a long period of 13 years, fully covered by our RV data. The minimum mass of the secondary is rather large, $0.5 \mathcal{M}_{\odot}$, and its lines are likely blended with those of the primary, reducing the RV amplitude. It is also an astrometric binary.

HIP 36758 (BD+39 1967, F8, 168 pc) might have a variable $\mathrm{RV}$, although our 11 measurements are not conclusive.

HIP 38195 (HD 63207, G 111-38, G5, 111 pc) is a threetier visual quadruple system with separations of $109^{\prime \prime}, 2$ '”2, and 0 '”084. It is metal-poor. One of our ten RVs deviates from the rest, suggesting variability. However, Latham et al. (2002) found a constant RV of $71.61 \mathrm{~km} \mathrm{~s}^{-1}$, so the existence of a spectroscopic subsystems is unlikely. The inner pair has an estimated period of $\sim 20$ years and should cause slow RV changes.

HIP 39681 (HD 66948, G5IV, 68 pc) is a single-lined binary with a long $4.7 \mathrm{yr}$ period. Astrometric orbit with similar period has been published by Goldin \& Makarov (2007). The large minimum secondary mass, $0.5 \mathcal{M}_{\odot}$, indicates that the RV amplitude could be reduced by line blending.

HIP 40253 (HD 68119, F5, $116 \mathrm{pc}$ ) is a single-lined binary with $P=808 \mathrm{~d}$, as well as an astrometric binary.

HIP 40724 (BD-14 2469, K5V, 35 pc) has only two RV measurements that differ by $2 \mathrm{~km} \mathrm{~s}^{-1}$; it is an astrometric binary.

$H D 71028$ is a distant $(480 \mathrm{pc})$ chromospherically active K0III giant for which we determine an orbit with $P=1130 \mathrm{~d}$.

HIP 42507 (BD-05 2603, K6V, 26 pc) has a large $\chi^{2} /(N-1)$ caused by one outlying measurement, hence the RV variability is not certain.

HIP 42550 (HD 73394) is a distant (600 pc) G5III giant. Its visual companion B (ES 209) at 57" separation is optical, with different PM and RV. Both stars were observed here, and we found that B has double lines. No orbit can be derived from our five spectra.
HIP 43820 (HD 75632, M1V, 11.6pc) is a visual binary on a $609 \mathrm{yr}$ orbit, currently at $3^{\prime \prime}$ separation. The RV of the brighter component A varies slowly over the 14 years of our monitoring, while its mean value differs slightly from the two $\mathrm{RVs}$ of the component $\mathrm{B}$. We therefore believe that it could be a triple system. Halbwachs et al. (2018) published nine pairs of RVs, splitting the CORAVEL correlation dips in two components. Combining these data with our RVs and with two RVs from Tokovinin \& Smekhov (2002), we can fit an orbit with $P=$ $1050 \mathrm{~d}, K_{1}=2.8 \mathrm{~km} \mathrm{~s}^{-1}, K_{2}=7.0 \mathrm{~km} \mathrm{~s}^{-1}$, and $\gamma=44.6 \mathrm{~km} \mathrm{~s}^{-1}$. The minimum masses are suspiciously small, 0.05 and $0.02 \mathcal{M}_{\odot}$, and therefore we prefer not to publish this orbit.

HIP 46383 (BD+40 2208, K4V, 32 pc) is a double-lined pair with $P=8.5 \mathrm{~d}$ and equal components, $q=0.99$. After the orbit was computed from our $12 \mathrm{RVs}$, the paper by Halbwachs et al. (2018) came to our attention. Here we fit the orbit to the combined set of $33 \mathrm{RVs}$. The small residuals of 0.38 and $0.43 \mathrm{~km} \mathrm{~s}^{-1}$ for the primary and secondary components, respectively, demonstrate the good quality of our RVs and the lack of substantial zero-point differences between the CORAVELs at Moletai and OHP and the VUES. Considering the physical companion at 56" (LEP 36), this is a triple system.

$B D-082689$ (M0V) shows an increasing RV over the 6 years of our monitoring. Simbad quotes only a crude parallax of $9.2 \pm$ 15.0 mas that places the star at 3 mag above the main sequence (see Fig. 1). This parallax is most likely inaccurate. Gaia DR2 provides no parallax because the star is a resolved 0 '!2 binary BEU 13 with an estimated period of $\sim 80 \mathrm{yr}$.

HIP 46926 (BD+16 1992, G0, $107 \mathrm{pc})$ is a triple system. The outer $33^{\prime \prime}$ pair is physical (common PM and parallax). We discovered the double-lined inner subsystem Aa,Ab with $P=3.1$ days and determined its orbit. Comparison of the RV amplitudes with the estimated masses implies a low orbital inclination of $15^{\circ}$.

HIP 47133 (M0V) is the secondary component $\mathrm{B}$ of a young multiple system belonging to the $\beta$ Pictoris moving group (Alonso-Floriano et al. 2015). The primary is HIP 47110 (HD 82939, G5V, 39 pc), at 162" separation from B. The two stars have common PM, RV, and parallax; the estimated period of A,B is $\sim 160 \mathrm{kyr}$. The component B was found to be a doublelined spectroscopic binary by Schlieder et al. (2012), but they have not provided its orbit. We observed the component B for almost 16 years and derive here an orbit with $P=4.39$ days. The residuals are quite large, $2.7 \mathrm{~km} \mathrm{~s}^{-1}$, partly because the star is faint and partly because of its chromospheric activity. The outstanding feature of this orbit is its large eccentricity of 0.47 . This pair is still in the phase of tidal orbit circularization. However, more observations are needed to confirm and improve the orbit.

HIP 47899 (MCC 554, K4V, 79 pc) is also a double-lined binary with a period of 146 days and a small but statistically significant orbital eccentricity. The mass ratio is $q=0.94$.

HIP $48346(\mathrm{BD}+382075, \mathrm{~K} 8,52 \mathrm{pc})$ is a double-lined binary with $P=79 \mathrm{~d}$, rather large eccentricity $e=0.7$, and no other known visual companions.

HIP 50156 (DK Leo, M0.7V, 23 pc) is a triple system composed of the 0 '. 1 visual binary A,B with an estimated period of $\sim 3 \mathrm{yr}$, also detected by astrometric acceleration, and the 73 day single-lined inner pair Aa,Ab found here. It is a variable star of BY Dra type. The system belongs to the $\beta$ Pictoris group according to several authors such as (Schlieder et al. 2012; Alonso-Floriano et al. 2015; Messina et al. 2017) and has an extensive bibliography.

HIP 50271 (BD+26 2062, G0, 173 pc) is a distant $\left(869^{\prime \prime}\right)$ optical companion to the nearby (37 pc) G0V star HIP 50355. 
Our observations lead to the single-lined orbit with $P=$ 47.5 days.

HIP 52021 (BD-05 3108, K8, 38 pc) has a variable RV, but our data do not suffice to find its orbit.

HIP 54002 (AB Crt, K3V, 31 pc) is a BY Dra-type variable star with variable RV, but no known spectroscopic orbit.

HIP 54094 (BD+54 1411, unknown spectral type, 47 pc) has the first deviant RV measurement which suggests its variability. Further monitoring is needed.

HIP 56229 (BD+41 2201, M0, 44 pc) is a double-lined binary. The 186 day spectroscopic orbit derived here from only seven RVs is not very secure. The star is an X-ray source. Astrometric acceleration was detected.

HIP 57058 (GJ 435.1, K4V, $31 \mathrm{pc}$ ) is a spectroscopic and acceleration binary for which we derive a preliminary singlelined orbit with $P=726 \mathrm{~d}$ by combining our RVs with those of Halbwachs et al. (2018). These authors found that the correlation dips are double and measured the RVs of the secondary component. The VUES profiles are double as well. We also measure the RVs of both components. This means that the CORAVEL RVs derived by fitting a single component are biased both in our data and in those of Halbwachs et al. when the RV difference is small. We use CORAVEL RVs with a low weight and disregard some of them. The period and estimated masses of $0.69 \mathcal{M}_{\odot}$ correspond to a semimajor axis of 57 mas. This star was resolved twice in 2018 by speckle interferometry at the Southern Astrophysical Research Telescope, SOAR, at similar separations and shows a fast orbital motion, in qualitative agreement with the two-year period (Tokovinin et al., in prep.).

The RVs of the secondary component do not vary in antiphase with the primary and therefore cannot be used to derive a double-lined orbit. Hypothetically, this is a triple system where the outer orbit has a small inclination (hence small $K_{1}$ ) and its secondary component contains a short-period subsystem. Spectroscopic monitoring with higher resolution and future astrometric orbits from Gaia and SOAR will clarify the architecture of this low-mass system.

$B D+442120(\mathrm{~F} 5,345 \mathrm{pc})$ is a distant triple system. The outer 6".8 pair ES 123 is physical, based on the common distances and RVs (the PMs are very small). Its secondary component B is a single-lined binary with $P=2.2$ days. We also observed the component $\mathrm{C}$ at $42^{\prime \prime}$ separation. Its $\mathrm{RV}$, as well as PM, are distinct, therefore the star $\mathrm{C}$ is unrelated (optical).

GQ Leo (TYC 870-798-1, K5Ve, $61 \mathrm{pc}$ ) has a slowly variable $\mathrm{RV}$. Its mean value of $-12.8 \mathrm{~km} \mathrm{~s}^{-1}$ differs from $-15.7 \pm 1.8 \mathrm{~km} \mathrm{~s}^{-1}$ measured by Gaia and from three RVs around $-11 \mathrm{~km} \mathrm{~s}^{-1}$ reported by Griffin (2005). An orbit with $P \approx 572 \mathrm{~d}$ and $K_{1}=$ $2.8 \mathrm{~km} \mathrm{~s}^{-1}$ can be fitted to all RVs. However, alternative periods are not excluded, and therefore we refrain from publishing this tentative orbit. The object is a 0 "'.25 pair MET 57Aa,Ab with an estimated period of $\sim 45$ years. Some RV variation could therefore be caused by this binary. The WDS lists another companion at $9^{\prime \prime} .5$, which is optical according to the Gaia astrometry.

HIP 57857 (G148-14, K0V, 54 pc) has a variable RV, but our data are not sufficient for the determination of its orbit. Astrometric acceleration is evident from comparison of the average PM of $(-339.3,-44.4)$ mas $^{-1}{ }^{-1}$ deduced from the difference of Hipparcos and Gaia positions with the "instantaneous"

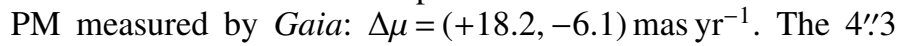
pair LEP 46 is physical according to Gaia PM and parallax, meaning that this is a triple system. The component $\mathrm{B}$ has an $\mathrm{RV}$ of $-14.3 \pm 3.3 \mathrm{~km} \mathrm{~s}^{-1}$ (Gaia) and its PM matches the average $\mathrm{PM}$ of $\mathrm{A}$.
HIP 57949 (MCC 622, M0.5Ve, 31 pc) also has a variable $\mathrm{RV}$, likely with a long period.

HIP 59000 (HD 105065, K5V, 23 pc) is a triple or quadruple system. The outer 183" binary (estimated period $~ 300 \mathrm{kyr}$ ) is physical. The main star is an acceleration binary. Our single RV measurement, $-8.2 \mathrm{~km} \mathrm{~s}^{-1}$, differs from the Gaia RV of $-29.2 \pm 13.6 \mathrm{~km} \mathrm{~s}^{-1}$. The large error of the latter also signals variability. Two mutually discordant RVs were measured by Maldonado et al. (2010). It is not clear whether the spectroscopic and astrometric subsystems are the same or distinct.

HIP 60433 (BD+21 2415, K4V, 40 pc) and HIP 60448 (MCC 654, K5V, 30 pc) both have variable RVs, but no orbits have been computed. The first is also an acceleration binary. Eighteen years of RV coverage is available for both stars.

HIP 61436 (GJ 9412, K5V, $30 \mathrm{pc}$ ) is a double-lined binary with $P=299 \mathrm{~d}$.

HIP 62505 (HD 111312, K2V, $26 \mathrm{pc}$ ) is a close visual and spectroscopic binary WSI 74 with a period of $2.66 \mathrm{yr}$. Its combined orbit was determined by Tokovinin (2017). Another visual companion at 2.77 was measured by HipParcos, but never confirmed; it is not spotted by Gaia and therefore remains questionable.

HIP 62755 was originally mis-identified with a nearby $\mathrm{K} 6 \mathrm{~V}$ dwarf MCC 679. The Gaia parallax of $0.81 \pm 0.03$ mas places HIP 62755 among giants. We determined an orbit with $P=$ 6.12 years from $11 \mathrm{RVs}$ covering a time period 8.2 years.

HIP 63253 (GJ 490, M0V, 21 pc) has an RV trend, and therefore the period is longer than 7 years. This is a $2+2$ quadruple system. The outer $16^{\prime \prime}$ pair has a period of $\sim 6 \mathrm{kyr}$. Both components were resolved into $00^{\prime \prime} 1$ pairs with estimated periods of a few years. The observed RV variation is most likely related to the subsystem Aa,Ab.

HIP 63816 (GJ 497, M0V, 16 pc) is a 1".6 visual binary WOR 23. We find its RV to be constant over a time span of 7 years.

HIP 63942 (BD+21 2486, K4V, 19 pc) has a constant RV. This is a visual binary HU 739 with an orbital period of 431 years and a semimajor axis of 2 ". 65 .

HIP 65012 (GJ 507B, M3V, $14 \mathrm{pc}$ ) is the secondary component B of HIP 65011 (at 17'.8 distance) which itself is a visual and spectroscopic pair with $P=200 \mathrm{~d}$. We measured $\mathrm{RV}(\mathrm{B})$ once and found $-5.2 \mathrm{~km} \mathrm{~s}^{-1}$; Gaia measured it at $-9.1 \pm$ $0.6 \mathrm{~km} \mathrm{~s}^{-1}$.

HIP 65026 (HD 115953, K0, 9 pc) is a remarkable triple system. The outer 1'.5 binary HU 644 has a good-quality visual orbit with a period of $49 \mathrm{yr}$. The inner subsystem $\mathrm{Aa}, \mathrm{Ab}$ is also resolved as CHR 193 at 0 '”. 1 . Here we determined its single-lined orbit with a period of $447 \mathrm{~d}$. A preliminary period of $450 \mathrm{~d}$ was announced by Beuzit et al. (2014). A preliminary combined orbit of the inner subsystem shows that the mutual inclination in this triple system is small.

HIP 65327 (HD 238224, K5V, 24 pc) was resolved by Hipparcos at 0.3 separation and $\Delta m=2.3 \mathrm{mag}$. We computed its single-lined orbit with $P=12.4$ years. However, the $\mathrm{RV}$ amplitude is likely reduced by line blending. A combined visual/spectroscopic orbit can be computed now. The WDS also mentions the wide CPM pair SHY 67 with a separation of 9:4, too wide to be a bound binary.

GJ $513(\mathrm{M} 3 \mathrm{~V}, 19 \mathrm{pc})$ has a slowly variable RV.

HIP 65887 (HD 117466, K0, $3 \mathrm{kpc}$ ) is a distant giant for which we provide a single-lined orbit with a 3.3 year period. Its semimajor axis should be $11 \mathrm{mas}$, and, indeed, the astrometric acceleration was detected by HiPPARcos. 
HIP 66290 (HD 118244, F5V, 38 pc) is a single-lined binary with a period of 5.4 years, as well as acceleration binary. The orbit is determined from $36 \mathrm{RVs}$ measured during 23 years.

HIP $67086(\mathrm{~K} 5,46 \mathrm{pc})$ is a 0 '!6 binary resolved by HIPPARcos (estimated period $\sim 100 \mathrm{yr}$ ) containing a spectroscopic subsystem. We measured RVs of both components with VUES and determined the orbit of the secondary subsystem $\mathrm{Ba}, \mathrm{Bb}$ with $P=41 \mathrm{~d}$.

$B D+262498$ is a G5 giant with a DR2 parallax of $1.40 \pm$ 0.03 mas. Our $31 \mathrm{RVs}$ measured over 18 years securely define the spectroscopic orbit with a period of 4.2 years. This orbit corresponds to the minimum secondary mass of $1 \mathcal{M}_{\odot}$. The secondary component could be a compact remnant. Gaia is expected to detect acceleration or deliver a full astrometric orbit.

HIP 67808 (BD+13 2721, K7V, 22 pc) is a 0 '.2 visual (and acceleration) binary with an estimated period of $\sim 10$ years discovered by Beuzit et al. (2014). Our six RVs measured over 7 years are probably constant, with one measurement deviating from the rest.

$B D+192735(\mathrm{~K} 2,37 \mathrm{pc})$ is a single-lined binary with a period of almost 10 years; 1.5 orbital cycles of its eccentric orbit are covered. Rotational modulation was measured by Kiraga (2012).

HIP 68801 (HD 123034, G5, 51 pc) is a double-lined binary with a circular 2.8 day orbit and a mass ratio $q=0.98$ (a twin). Nordström et al. (2004) discovered the RV variability but provided no orbit.

HIP 69549 (HD 124605, G0, 85 pc) is a double-lined pair with $P=6 \mathrm{~d}$ and nearly equal components, $q=0.98$. The interferometric 0 '.08 pair TOK 723 with $\Delta I=2.4$ mag remains unconfirmed and could be spurious; its parameters are similar to the optical ghosts reported in the discovery paper by Tokovinin et al. (2018). If it were a triple system, the outer period would be around $\sim 10 \mathrm{yr}$. However, we do not see any modulation of the center-of-mass velocity over the 19 years covered by our data.

HIP 72508 (HR 5537, F5IV, 52 pc) has double lines but the period remains unknown. The WDS companion B at $15^{\prime \prime}$ is optical according to its Gaia astrometry. However, Gaia detected another faint $(G=17.42 \mathrm{mag})$ star at 9'.2 separation with similar parallax and PM, therefore this system is at least triple.

$B D+492364$ is a giant according to the Gaia parallax of $0.97 \pm 0.03$ mas. Its RV shows a trend and has changed by $5 \mathrm{~km} \mathrm{~s}^{-1}$. The orbital period is longer than 29.5 years covered by our data.

HIP 76941 (MCC 316, K5V, 50 pc) is a single-lined binary with $P=267 \mathrm{~d}$.

HIP $77141(\mathrm{BD}+362641, \mathrm{~K} 4 / 5 \mathrm{~V}, 54 \mathrm{pc})$ has a well-defined orbit with $P=17.3 \mathrm{~d}$ and a very large (for this period) eccentricity of $0.84 \pm 0.15$. Better coverage near periastron of this orbit is needed to constrain the eccentricity.

HIP $78158(\mathrm{~K} 5 \mathrm{~V}, 52 \mathrm{pc})$ is a triple system that consists of the wide 187" physical pair A,B (LDS 983) and the singlelined spectroscopic subsystem $\mathrm{Aa}, \mathrm{Ab}$ discovered here. Its orbital period is $322 \mathrm{~d}$. We noted secondary dips in some spectra, but their RVs do not match the orbit.

HIP 79796 (BD+55 1823, CR Dra, M5.6V, 20 pc) is a lowmass flare star and an interferometric binary BLA 3, for which an orbit with a period of $4.04 \mathrm{yr}$ was computed by Tamazian et al. (2008). We see double lines, but their RVs do not match the visual orbit. A tentative RV curve with a period of $1.57 \mathrm{yr}$ is plotted in Fig. A.1. Shkolnik et al. (2010) obtained two double-lined spectra and determined that the period is less than $530 \mathrm{~d}$. More work is needed to reconcile RVs with position measurements and hopefully to compute the combined orbit.

HIP 80751 (BD+243014, K5V, 32 pc) has a variable RV measured over 18 years. We determined a tentative orbit with $P=$ $14.4 \mathrm{yr}$, but new measurements and a better coverage are needed for its confirmation. Astrometric acceleration was detected.

$B D+521986(\mathrm{~K} 8,44 \mathrm{pc})$ is a 5".6 visual binary ES 968 (estimated period $\sim 3 \mathrm{kyr}$ ). The RV of the component $\mathrm{A}$, measured 13 times is certainly variable with a long period. The RV of $\mathrm{B}$ was measured on only one night and agrees with the mean RV of A.

HIP 82506 (HD 152342, F4III, 67 pc) has a variable RV, but not enough data are available for orbit calculation. Is is also an astrometric binary.

$B D+611678 C$ (GJ 685, M1V, $14 \mathrm{pc}$ ) is the distant $\left(738^{\prime \prime}\right)$ component to the visual pair A,B (GJ 684, HIP 86036, G0V) which has a period of $76 \mathrm{yr}$ and the corresponding single-lined spectroscopic orbit. We monitored RV of the component $\mathrm{C}$ and found it to be constant, agreeing with the RV of A. The same conclusion was reached by Tokovinin (1992).

HIP 90274 (HD 170527, K0, 175 pc) is a giant observed over 16 years. The spectrum has blended double lines, but no orbit is derived yet.

HIP 91043 (HD 171488, V889 Her, G2V, 35 pc) has a double-lined spectrum and no spectroscopic orbit despite our 39 observations, mostly with unresolved CORAVEL dips. Several faint companions are listed in the WDS, but none of those are confirmed as physical.

HIP 92952 (G 229-18, M0V, 46 pc) is a quadruple system. The outer pair A,B (GIC 154) has a separation of $119^{\prime \prime}$ and an estimated period of $\sim 300 \mathrm{kyr}$. The component $\mathrm{A}$ is a 0 ". 4 visual binary $\mathrm{Aa}, \mathrm{Ab}$ resolved by HipParcos that has not been measured since; its estimated period is $\sim 70$ years. We see double lines in the spectrum. Stationary lines correspond to the visual secondary $\mathrm{Ab}$ and the moving lines to the primary $\mathrm{Aa}$, which is a spectroscopic binary Aa1,Aa2 with a period of $8 \mathrm{~d}$. The eccentricity of the spectroscopic orbit is small, but statistically significant: $e=0.044 \pm 0.007$. The RV of the star Ab is about $-15 \mathrm{~km} \mathrm{~s}^{-1}$. Its measurements are not accurate owing to blending with the lines of Aa1.

HIP 94557 (G 185-12, M4.5V, 18 pc) is the brighter component of the wide visual binary WDS J19147+1918 (LDS 2020, $41^{\prime \prime}$ ). Our three RVs show variability. Shkolnik et al. (2012) measured a very different RV of $-80.9 \mathrm{~km} \mathrm{~s}^{-1}$, quoted in Simbad.

HIP 94622 (GJ 751, M0, 29 pc) has a variable RV, and some spectra have double lines. The RV variability was also noted by Tokovinin (1992). This is a HIPPARcos binary with 0'!2 separation and an estimated period of $\sim 10 \mathrm{yr}$. It is not clear whether the visual and spectroscopic systems correspond to the same pair or this is a triple system. $17.3 \mathrm{~d}$

$B D+77767(\mathrm{~K} 8,41 \mathrm{pc})$ is a single-lined binary with $P=$

HIP 99969 (BD+06 4489, K4V, 44 pc) has double lines, but we are not yet able to determine its orbit.

HIP 101941 (HD 196928, K4III, $380 \mathrm{pc}$ ) is a single-lined binary with $P=2.8 \mathrm{yr}$, as well as an astrometric binary.

HIP 102300 (M0Ve, $21 \mathrm{pc}$ ) has a variable RV. The period is longer than the $8 \mathrm{yr}$ covered by our data.

HIP 102320 (HD 335007, K5, $42 \mathrm{pc}$ ) belongs to a triple system where the outer 4!.4 pair ES 366 has a period of the order of $\sim 2 \mathrm{kyr}$ and $\Delta m=3 \mathrm{mag}$. The main component $\mathrm{A}$ is a double-lined twin binary with $P=21 \mathrm{~d}$ and equal components, $q=0.97$. The orbital inclination of the spectroscopic pair is about $55^{\circ}$. 
HIP 102718 (BD+04 4551, F7Vw, $103 \mathrm{pc}$ ) is the primary component of WDS J20488+0512, a 6"'6 pair. Its RV is likely constant.

HIP 103375 (HD 235405, G0, $132 \mathrm{pc}$ ) belongs to WDS J20566+5250 (ADS 14465, A 1437, separation 1'!3). Its RV is likely constant.

HIP 104994 (BD+28 4035, G5, 143 pc) is a triple-lined system. The outer 0 "' 19 binary was first resolved by HIPPARCos and has not been measured since; its period is $\sim 100 \mathrm{yr}$. Stationary lines in the spectrum belong to the visual secondary B with RV of $-54 \mathrm{~km} \mathrm{~s}^{-1}$. The pair of moving lines corresponds to the subsystem $\mathrm{Aa}, \mathrm{Ab}$ with $P=52 \mathrm{~d}$ and systemic velocity of $-46.8 \mathrm{~km} \mathrm{~s}^{-1}$. Its inclination is about $52^{\circ}$.

HIP 105504 (HD 358435, K7, 42 pc) definitely has a variable $\mathrm{RV}$, but not enough data for an orbit. Its astrometric acceleration was detected.

$B D+473439(\mathrm{~K} 0,115 \mathrm{pc})$ is an evolved star in the visual triple system ADS 15052, with separations of $1{ }^{\prime \prime} 1$ and $0 \prime 2.2$. The spectra have double lines, but the spectroscopic orbit remains to be determined. Either there is an additional spectroscopic subsystem, or the inner binary is going through the periastron of its eccentric orbit (its estimated period is $\sim 80$ years). The fact that Gaia DR2 measured the parallax favors the latter scenario because partially resolved sources do not have parallaxes in DR2.

HIP 110291 (HD 212029, G0, 62 pc) is a single-lined binary with a period of $2.1 \mathrm{yr}$. A similar period was determined by $\mathrm{D}$. Latham (2012, priv. comm.), while two astrometric orbits with periods of 2.07 and $2.17 \mathrm{yr}$ and large eccentricities were computed by Goldin \& Makarov (2006). The WDS lists several optical companions.

HIP 110526 (GJ 826, M3.0V, $15 \mathrm{pc}$ ) has a constant RV over the 8 years of our monitoring. It is a visual binary WOR 11 with $P=130$ yr and semimajor axis $11^{\prime \prime} 61$.

HIP 110978 (HD 213054, K2III, 800 pc) may have a mild $\mathrm{RV}$ trend over 34 days. It has astrometric acceleration.

HIP 111685 (BD+38 4818, M0Ve, $24 \mathrm{pc}$ ) is the main component of the triple system. The faint ( $V=21.2 \mathrm{mag}$ ) outer component $\mathrm{C}$ is at 33".4 distance from the main star, with common PM and parallax. The inner pair A,B, first resolved by HiPPARcos, has a well-defined visual orbit with $P=16 \mathrm{yr}$. We fitted our RVs and all available positional measurements to a combined orbit and provide its spectroscopic elements here.

HIP 111942 (GJ 870, K8V, $31 \mathrm{pc}$ ) is a single-lined binary with $P=375$ days. The orbit derived from our $20 \mathrm{RVs}$ is very similar to the orbit published independently by Halbwachs et al. (2018) and based on 25 CORAVEL RVs. Here we list orbital elements fitted to the combined data, with a global rms residual of $0.32 \mathrm{~km} \mathrm{~s}^{-1}$.

HIP 112040 (BD+18 5029, M0V, 31 pc) has a variable RV with a period longer than $15 \mathrm{yr}$, as well as astrometric acceleration.

HIP 112268 (BD+16 4806, K6V, 50 pc) has a variable RV. We computed an uncertain orbit with $P=4.35 \mathrm{yr}$ which is not publishable until better coverage is obtained.

HIP 112523 (MCC 851, K5V, 37 pc) has a preliminary orbit with $P=8.9 \mathrm{yr}$ derived from $13 \mathrm{RV}$ spanning a period of $15 \mathrm{yr}$. The eccentricity was fixed in the orbit fitting. Astrometric acceleration was detected.

HIP 116003 (GJ 1284, M2Ve, 16 pc) is a flare star and an $\mathrm{X}$-ray source. As our two RVs are very different from each other, this object must be a fast spectroscopic binary. A variable RV was noted by Gizis et al. (2002).

$B D+661664(\mathrm{G} 5,90 \mathrm{pc})$ is a single-lined binary with a circular three-day orbit. Fast synchronous rotation is responsible for its high chromospheric activity.
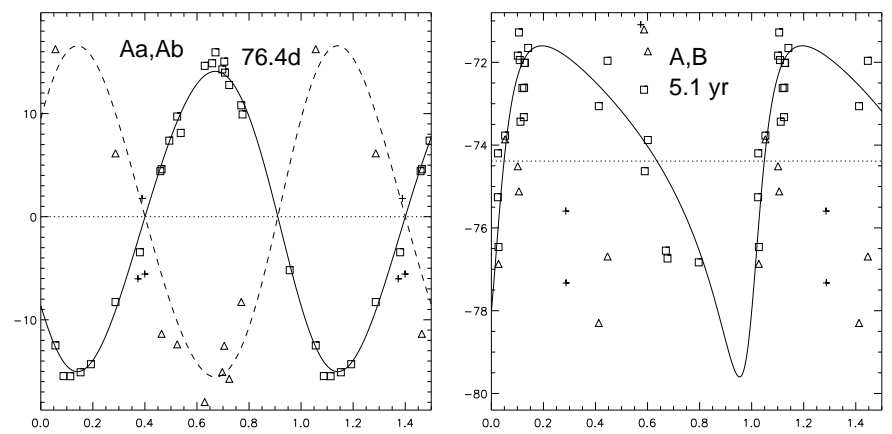

Fig. 3. RV curves of HIP 13460. Left: inner subsystem, $P=76.4 \mathrm{~d}$. Right: outer subsystem, $P=5.1$ yr. Radial velocities derived from blended dips are plotted as crosses and are given small weights in the orbit fit.

HIP 118212 (GJ 913, K7V, 17 pc) is a single-lined binary with a period of $872 \mathrm{~d}(2.4 \mathrm{yr})$. It is also an acceleration binary and suspected nonsingle star in Hipparcos. One interferometric resolution at 62 mas with $\Delta I=1.4$ mag was reported by Balega et al. (2007). The astrometric orbit by Goldin \& Makarov (2006) with $P=885 \mathrm{~d}$ and $e=0.56$ is similar to our spectroscopic orbit. However, their revised parallax of 67 mas is not confirmed by Gaia.

\subsection{The triple system HIP 13460}

This star, also known as BD+60 585 and GJ 3185, is a $\mathrm{K} 3 \mathrm{~V}$ dwarf at $39 \mathrm{pc}$ from the Sun. The spectrum is double-lined, and the period of $76 \mathrm{~d}$ is readily found. However, all $30 \mathrm{RV}$ s cannot be fitted by the common elements, leaving residuals of 1.6 and $2.0 \mathrm{~km} \mathrm{~s}^{-1}$ for $\mathrm{Aa}$ and $\mathrm{Ab}$, respectively. Individual fits to the RVs measured with CORAVEL and VUES are better, but result in slightly different elements.

All RVs can be better modeled by assuming that the pair $\mathrm{Aa}, \mathrm{Ab}$ moves slowly on an outer orbit. Astrometric acceleration reported by Makarov \& Kaplan (2005) and confirmed by Gaia supports the triple-star hypothesis. We fitted the long-period orbit with $K_{1}=4 \mathrm{~km} \mathrm{~s}^{-1}$ using orbit3.pro (Fig. 3). The rms residuals to the triple-star solution are $0.88 \mathrm{~km} \mathrm{~s}^{-1}$ for Aa and $3.23 \mathrm{~km} \mathrm{~s}^{-1}$ for $\mathrm{Ab}$. The component $\mathrm{Ab}$ may contain a shortperiod subsystem.

The masses of $\mathrm{Aa}$ and $\mathrm{Ab}$ estimated from the absolute magnitudes and the mass ratio are 0.78 and $0.71 \mathcal{M}_{\odot}$. Comparison with the minimum masses leads to the inclination $i_{\mathrm{Aa}, \mathrm{Ab}}=32^{\circ}$. The minimum mass of the tertiary component $\mathrm{B}$ is $0.29 \mathcal{M}_{\odot}$. Although the semimajor axis of the outer orbit computed from the period and mass sum is 92 mas, there is little hope of resolving A,B directly owing to the expected faintness of B. On the other hand, Gaia can provide astrometric orbits for both inner and outer systems. To do so, an initial guess of the orbital periods and other parameters will likely be needed, and our work provides these parameters. It is unlikely that Gaia astrometry of this complex system can be interpreted correctly from its pipeline alone, without additional inputs.

\section{Summary}

The main results of this work are as follows.

- A large set of RV measurements spanning three decades.

- Determination of 57 spectroscopic orbits, 53 of those for the first time. 


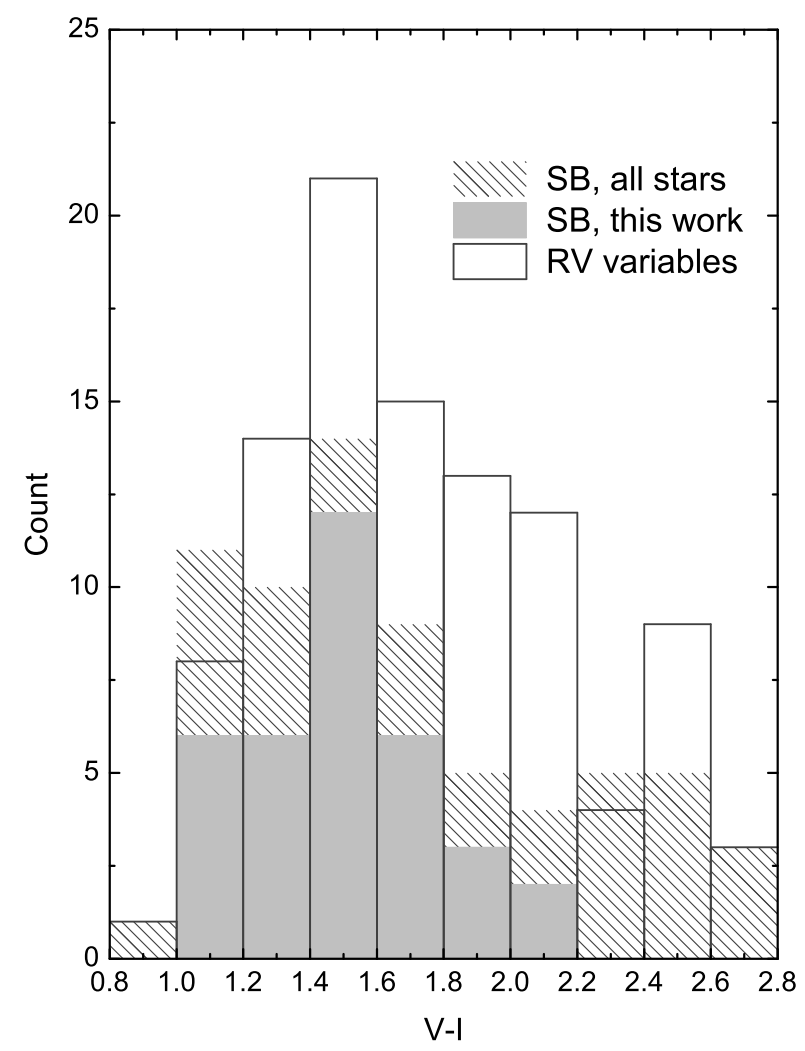

Fig. 4. Histogram of the number of spectroscopic binaries and stars with variable RVs in the sample of K- and M-dwarfs of Sperauskas et al. (2016).

- Discovery of 20 new nearby hierarchical systems.

- Discovery of interesting stellar systems. For example, in the young triple system HIP 47110, the inner orbit with $P=$ $4.4 \mathrm{~d}$ and $e=0.47$ is apparently still circularizing.

Most orbits presented here refer to nearby K- and M-type dwarfs and result from long-term RV monitoring. Our sample includes 857 stars from the McCormick catalog and 188 stars from the Gliese catalog. Observational data on this sample are given in Table 4 of Sperauskas et al. (2016). A total of 67 spectroscopic orbits are known for these stars, including 35 determined here. In addition, there are 70 stars with variable RVs without orbital elements identified from our data, from the literature (e.g. Halbwachs et al. 2018), or by comparing our RVs with those from Gaia DR2. The latter group contains 30 objects where the $\mathrm{RV}$ difference exceeds $2.5 \mathrm{~km} \mathrm{~s}^{-1}(3 \sigma)$. For stars with constant $\mathrm{RV}$, the mean RV difference between our CORAVEL and Gaia $\mathrm{RVs}$ is $0.21 \mathrm{~km} \mathrm{~s}^{-1}$ with the rms scatter of $\sigma=0.74 \mathrm{~km} \mathrm{~s}^{-1}$. Figure 4 illustrates the contribution of this work to the census of spectroscopic binaries among K- and M-dwarfs.

We continue observations of the identified RV variables with the VUES spectrograph in order to detect double-lined spectroscopic binaries among them and to calculate their orbital parameters. Stars of this type may not be recognized by Gaia due to the relatively low resolution of its spectrometer ${ }^{3}$.
Acknowledgements. It is a pleasure to thank an anonymous referee for careful reading and valuable comments. We used the Simbad service operated by the Centre des Données Stellaires (Strasbourg, France). This work also made use of data from the European Space Agency (ESA) mission Gaia, processed by the Gaia Data Processing and Analysis Consortium (DPAC; https://www . cosmos.esa.int/web/gaia/dpac/consortium). Funding for the DPAC has been provided by national institutions, in particular the institutions participating in the Gaia Multilateral Agreement.

\section{References}

Albareti, F. D., Allende Prieto, C., \& Almeida, A. 2017, ApJS, 233, 48 Alonso-Floriano, F. J., Caballero, J. A., Cortès-Contreras, M., Solano, E., \& Montes, D. 2015, A\&A, 583, 85

Balega, I. I., Balega, Y. Y., Maksimov, A. F., et al. 2007, Astrophys. Bull., 62, 339

Baranne, A., Mayor, M., \& Poncet, J. L. 1979, Vistas Astron., 23, 279

Baroch, D., Morales, J. C., Ribas, I., et al. 2018, A\&A, 619, 32

Beuzit, J.-L., Ségransan, D., Forveille, T., et al. 2014, A\&A, 425, 997

Bressan, A., Marigo, P., Girardi, L., et al. 2012, MNRAS, 427, 127

Chanamé, J., \& Ramirez, I. 2012, ApJ, 746, 102

Fekel, F. C., \& Marschall, L. A. 1991, AJ, 102, 1439

Gaia Collaboration (Brown, A. G. A., et al.) 2018, A\&A, 595, A1

Gizis, J. E., Reid, I. N., \& Hawley, S. L. 2002, AJ, 123, 3356

Gliese, W., \& Jahreiß, H. 1991, The Astronomical Data Center CD-ROM: Selected Astronomical Catalogs (Greenbelt: Goddard Space Flight Center), I

Goldin, A., \& Makarov, V. V. 2006, ApJS, 166, 341

Goldin, A., \& Makarov, V. V. 2007, ApJS, 173, 137

Griffin, R. F. 2005, Observatory, 125, 323

Halbwachs, J. L., Mayor, M., Udry, S., \& Arenou, F. 2003, A\&A, 397, 159

Halbwachs, J. L., Mayor, M., \& Udry, S. 2018, A\&A, 619, 81

Janson, M., Durkan, S., Hippler, S., et al. 2017, A\&A, 599, 70

Jurgenson, C., Fischer, D., McCracken, T., et al. 2016, JAI, 5, 500033

Kiraga, M. 2012, Acta Astron., 62, 67

Latham, D. W., Stefanik, R. P., Torres, G., et al. 2002, AJ, 124, 1144

Makarov, V. V., \& Kaplan, G. H. 2005, AJ, 129, 2420

Maldonado, J., Martinez-Arnaiz, R. M., Eiroa, C., Montes, D., \& Montesinos, B. 2010, A\&A, 521, 12

Malkov, O. Y., Oblak, E., Snegireva, E. A., \& Torra, J. 2006, A\&A, 446, 785

Mason, B. D., Wycoff, G. L., Hartkopf, W. I., Douglass, G. G., \& Worley, C. E. 2001, AJ, 122, 3466 (WDS)

Messina, S., Lanzafam, A. C., Malo, L., et al. 2017, A\&A, 607, 3

Moe, M., Kratte, K. M., \& Badenes, C. 2019, ApJ, 875, 61

Montes, D., González-Peinado, R., Tabernero, H. M., et al. 2018, MNRAS, 479, 1332

Niedever, D. L., Marcy, G. W., Butler, R. P., Debra, A., \& Vogt, S. S. 2002, ApJS, 141,503

Nordström, B., Mayor, M., Andersen, J., et al. 2004, A\&A, 418, 989

Schlieder, J. E., Lépine, S., \& Simon, M. 2012, AJ, 144, 109

Shkolnik, E. L., Hebb, L., Liu, M., Reid, I. N., \& Collier, C. A. 2010, ApJ, 716, 1522

Shkolnik, E. L., Anglada-Escude, G., Liu, M., et al. 2012, ApJ, 758, 56

Sperauskas, J., Bartašiute, S., Boyle, R. P., et al. 2016, A\&A, 596, 116

Steinmetz, M., Zwitter, T., Siebert, A., et al. 2006, AJ, 132, 1645

Tamazian, V. S., Docobo, J. A., Balega, Y. Y., et al. 2008, AJ, 136, 974

Tokovinin, A. 2018, ApJS, 235, 6

Tokovinin, A. A. 1992, A\&A, 256, 121

Tokovinin, A. A. 2014, AJ, 147, 87

Tokovinin, A. A. 2017, AJ, 154, 110

Tokovinin, A., \& Latham, D. W. 2017, ApJ, 838, 54

Tokovinin, A. A., \& Smekhov, M. G. 2002, A\&A, 382, 118

Tokovinin, A., Mason, B. D., Hartkopf, W. I., Mendez, R. A., \& Horch, E. 2018, AJ, 155, 235

Upgren, A. R., \& Weis, E. W. 1989, in Star Catalogues: A Centennial Tribute to A. N. Vyssotsky, eds. A. G. D. Philip, \& A. R. Upgren, 19

Zhao, G., Zhao, Y.-H., Chu, Y.-Q., Jing, Y.-P., \& Deng, L.-C. 2012, RAA, 12, 723

3 https://www.cosmos. esa.int/gaia 


\section{Appendix A: Additional material}
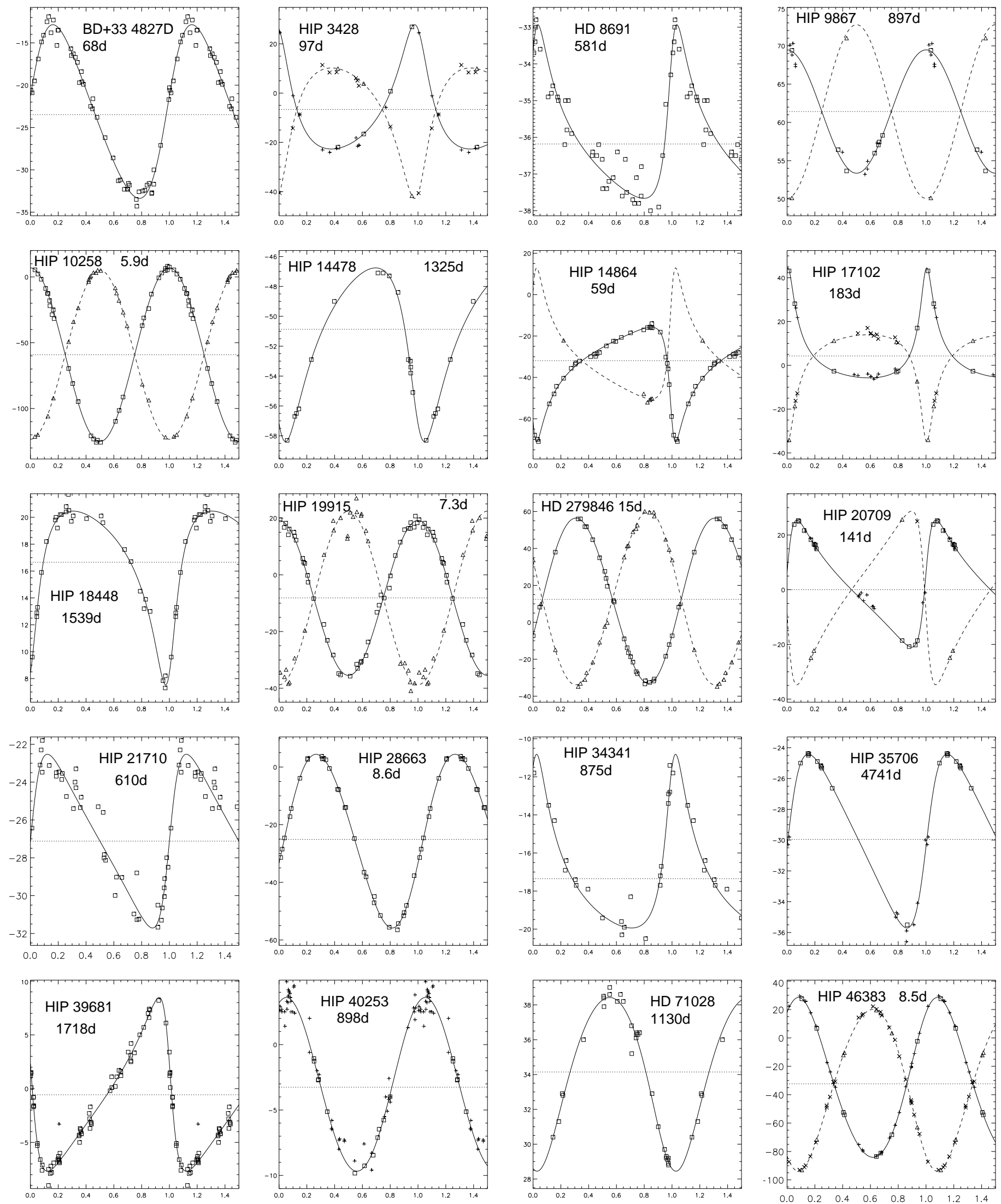

Fig. A.1. RV curves. Object names and approximate periods are indicated. In each plot, the horizontal axis is the orbital phase, the vertical axis is the $\mathrm{RV}$ in $\mathrm{km} \mathrm{s}^{-1}$. The RV curves of the primary and secondary components are plotted in full and dashed lines, respectively, while the squares and triangles denote the measurements. In some plots, crosses denote RVs with reduced weights. 
J. Sperauskas et al.: Spectroscopic orbits of nearby stars
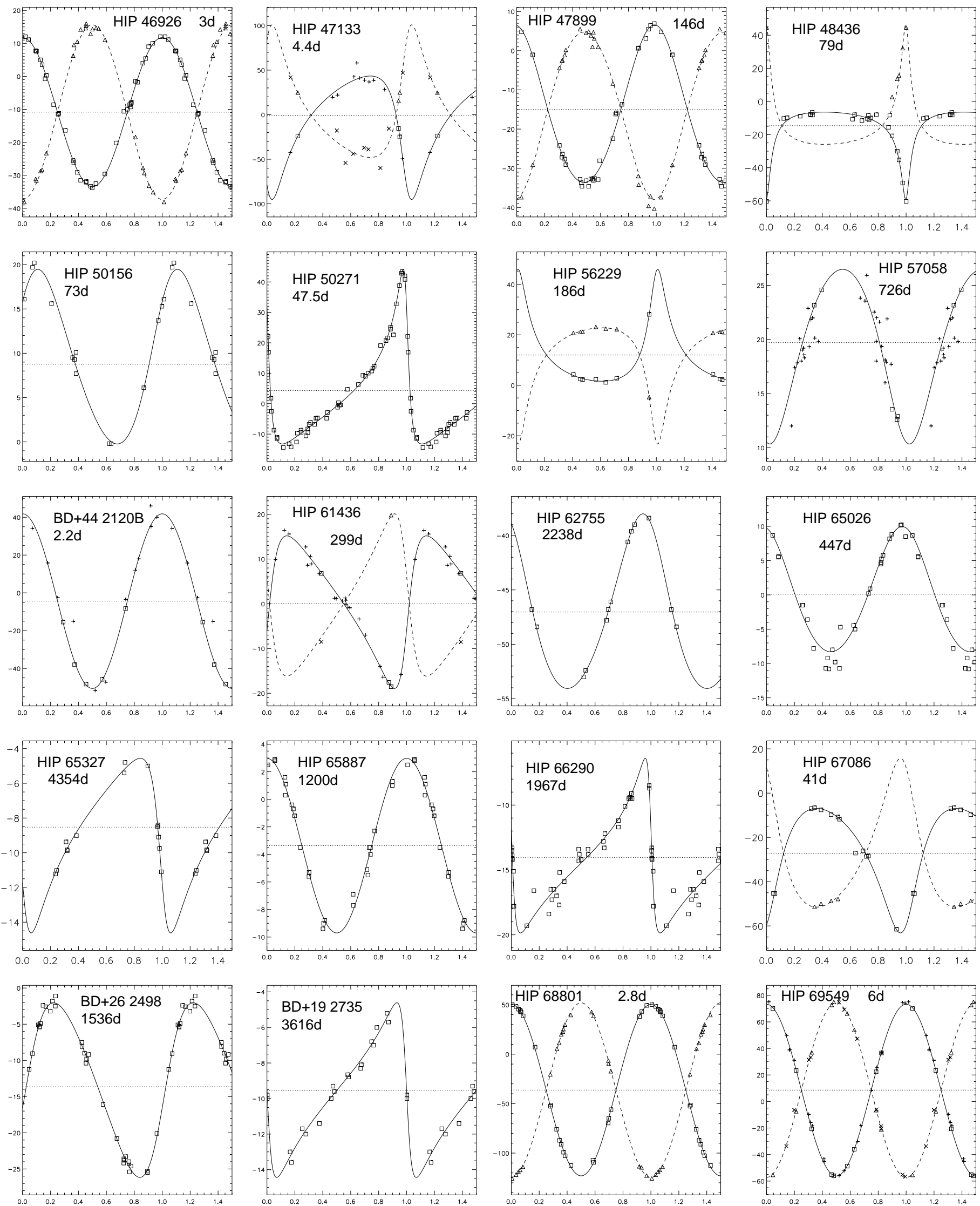

Fig. A.1. continued. 
A\&A 626, A31 (2019)
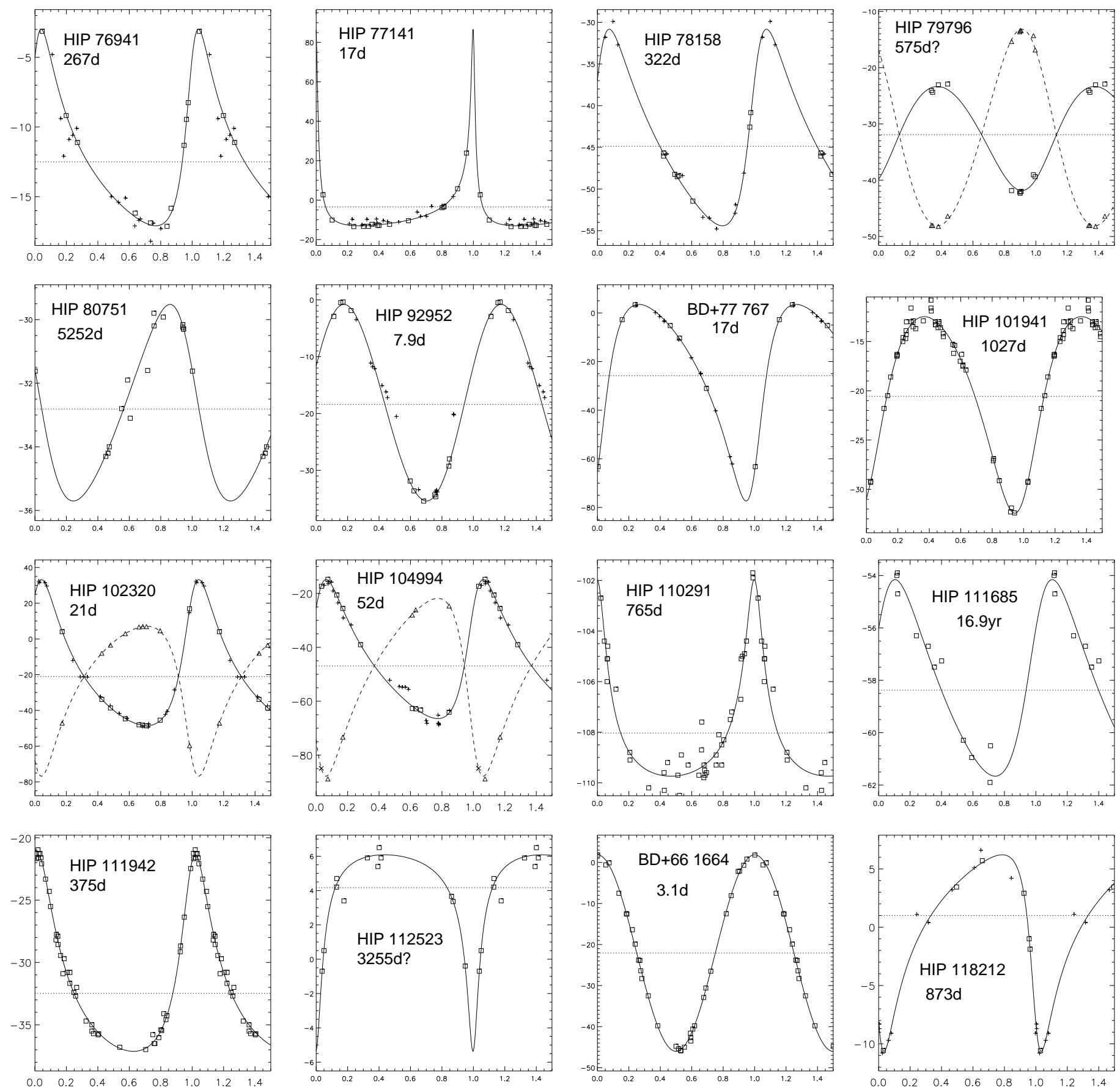

Fig. A.1. continued. 
Table A.1. Object list.

\begin{tabular}{|c|c|c|c|c|c|c|c|c|c|c|c|}
\hline Name & \multicolumn{2}{|c|}{ J2000 } & $\begin{array}{c}V \\
(\mathrm{mag})\end{array}$ & $\begin{array}{c}\varpi \\
(\mathrm{mas})\end{array}$ & $\begin{array}{l}\text { Sp. } \\
\text { type }\end{array}$ & Type & $N$ & $\begin{array}{l}\Delta T \\
\text { (d) }\end{array}$ & $\begin{array}{c}\langle\mathrm{RV}\rangle \\
\left(\mathrm{km} \mathrm{s}^{-1}\right)\end{array}$ & $\begin{array}{c}\mathrm{rms} \\
\left(\mathrm{km} \mathrm{s}^{-1}\right)\end{array}$ & $\chi^{2} /(N-1)$ \\
\hline HIP 96 & 000113.19 & +1358 30.3 & 10.59 & 23.25 & M0.5 & V & 7 & 2555 & -7.93 & 1.49 & 23.6 \\
\hline $\mathrm{BD}+13$ 5195B & 000112.87 & +1358 19.7 & 11.12 & 27.77 & M1 & V & 5 & 2541 & -10.15 & 0.82 & 10.4 \\
\hline HIP 374 & 000440.08 & +341554.4 & 7.08 & 5.64 & K0 & $\mathrm{C}$ & 4 & 6231 & -24.02 & 0.35 & 1.8 \\
\hline HIP 375 & 000440.19 & +341619.8 & 10.13 & 1.00 & - & $\mathrm{C}$ & 4 & 6242 & -9.17 & 0.24 & 0.9 \\
\hline $\mathrm{BD}+33$ 4827D & 000433.54 & +341503.5 & 10.55 & 4.56 & F9 & S1 & 49 & 6242 & -23.48 & $\ldots$ & $\ldots$ \\
\hline HIP 1412 & 001740.90 & -084056.2 & 10.95 & 31.57 & $\mathrm{~K} 7 \mathrm{~V}$ & $\mathrm{~V}$ & 6 & 1479 & 15.73 & 14.41 & 99.0 \\
\hline HIP 3428 & 004341.42 & +235307.1 & 10.97 & 22.18 & $\mathrm{~K} 7$ & S2 & 16 & 2168 & -6.65 & & \\
\hline HIP 5110 & 010529.92 & +152324.2 & 9.17 & 36.65 & $\mathrm{~K} 3.5 \mathrm{~V}$ & $\mathrm{C}$ & 4 & 2915 & -5.72 & 0.35 & 2.4 \\
\hline HD 6440B & 010529.76 & +152315.5 & 9.93 & 37.41 & K8V & $\mathrm{V}$ & 8 & 2915 & -3.22 & 1.23 & 12.7 \\
\hline HD 8691 & 012609.28 & +315452.6 & 9.24 & 19.96 & G0 & S1 & 42 & 5440 & -36.18 & $\ldots$ & $\ldots$ \\
\hline HIP 9867 & 020657.21 & +451104.1 & 10.24 & 51.47 & M0V & $\mathrm{S} 2$ & 17 & 5479 & 61.42 & $\ldots$ & $\ldots$ \\
\hline HIP 10258 & 021157.98 & +042141.8 & 9.72 & 21.36 & K5 & $\mathrm{S} 2$ & 30 & 4742 & -59.15 & & $\ldots$ \\
\hline $\mathrm{BD}+49646$ & 022233.89 & +503336.9 & 9.65 & 18.91 & - & $\mathrm{s} 2$ & 8 & 4711 & -10.00 & $\ldots$ & $\ldots$ \\
\hline HIP 11437 & 022729.25 & +305824.6 & 10.12 & 24.36 & K7V & $\mathrm{C}$ & 10 & 5884 & 5.98 & 0.46 & 1.8 \\
\hline HIP 12787 & 024421.36 & +105741.3 & 11.10 & 20.51 & MoVe & $\mathrm{s} 2$ & 12 & 2970 & 4.20 & & $\ldots$ \\
\hline HIP 13398 & 025225.03 & +265829.9 & 11.05 & 42.67 & $\mathrm{M} 2 \mathrm{~V}$ & $\mathrm{~V}$ ? & 13 & 5459 & -11.59 & 0.74 & 3.7 \\
\hline HIP 13460 & 025318.50 & +605111.7 & 9.20 & 25.75 & $\mathrm{~K} 3 \mathrm{~V}$ & $\mathrm{~S} 2$ & 30 & 2965 & -73.61 & & \\
\hline HIP 14478 & 030651.34 & +402133.5 & 9.63 & 37.02 & K6 & $\mathrm{S} 1$ & 15 & 2917 & -50.87 & $\ldots$ & $\ldots$ \\
\hline HIP 14669 & 030930.79 & +454357.9 & 10.17 & 57.11 & $\mathrm{M} 2 \mathrm{~V}$ & $\mathrm{~V}$ & 9 & 2629 & -4.51 & 1.99 & 35.2 \\
\hline HIP 14864 & 031156.83 & +613113.0 & 10.05 & 39.82 & M0Ve & S2 & 33 & 3023 & -32.10 & $\ldots$ & $\ldots$ \\
\hline $\mathrm{BD}+03480$ & 032814.92 & +040947.4 & 9.49 & 11.99 & G0 & $\mathrm{C}$ & 11 & 4764 & 12.66 & 0.57 & 1.1 \\
\hline HIP 17102 & 033948.96 & +332824.3 & 9.05 & 25.49 & $\mathrm{~K} 2$ & $\mathrm{~S} 2$ & 23 & 2777 & 4.27 & $\ldots$ & $\ldots$ \\
\hline GJ 3248 & 034838.24 & +733235.3 & 11.32 & 62.71 & M1V & $\mathrm{V}$ ? & 11 & 2971 & -8.08 & 0.78 & 5.0 \\
\hline HIP 18448 & 035636.22 & +695055.9 & 9.33 & 6.71 & K0 & $\mathrm{S} 1$ & 29 & 6014 & 16.66 & $\ldots$ & $\ldots$ \\
\hline HIP 19410 & 040926.37 & -144154.1 & 10.61 & 24.67 & $\mathrm{~K} 5 \mathrm{~V}$ & $\mathrm{~V}$ & 3 & 4350 & 6.34 & 7.97 & 99.0 \\
\hline HIP 19915 & 041619.83 & +364402.8 & 8.98 & 6.01 & F8 & S2 & 41 & 10496 & -8.16 & $\ldots$ & $\ldots$ \\
\hline HD 279846 & 042609.68 & +340934.2 & 10.50 & 12.14 & $\mathrm{~K} 2$ & $\mathrm{~S} 2$ & 28 & 6089 & 12.43 & $\ldots$ & $\ldots$ \\
\hline HIP 20709 & 042615.05 & +344257.2 & 8.29 & 7.60 & F5 & $\mathrm{S} 2$ & 27 & 4743 & 44.76 & $\ldots$ & $\ldots$ \\
\hline HIP 21710 & 043942.61 & +095219.5 & 9.19 & 36.80 & $\mathrm{~K} 2$ & $\mathrm{~S} 1$ & 16 & 1340 & -25.93 & $\ldots$ & $\ldots$ \\
\hline HIP 21845 & 044147.25 & +283935.9 & 8.85 & 8.70 & $\mathrm{~F} 8$ & $\mathrm{~V} ?$ & 11 & 5191 & 11.20 & 0.59 & 2.3 \\
\hline HIP 23550 & 050351.96 & +245822.1 & 7.43 & 13.69 & G8V & $\mathrm{V}$ & 16 & 3271 & 54.18 & 1.18 & 10.6 \\
\hline HIP 24488 & 051515.46 & +471014.6 & 6.92 & 8.73 & G5III & $\mathrm{s} 2$ & 10 & 7146 & 24.42 & $\ldots$ & $\ldots$ \\
\hline GJ 220 & 055314.04 & +241532.9 & 10.82 & 51.50 & $\mathrm{M} 2.0 \mathrm{~V}$ & V & 13 & 6089 & 26.45 & 1.75 & 28.2 \\
\hline HIP 28663 & 060308.64 & +142154.4 & 8.31 & 9.74 & F4IV & S1 & 30 & 1722 & -25.10 & $\ldots$ & $\ldots$ \\
\hline HIP 29295 & 061034.62 & -215152.7 & 8.12 & 173.70 & M1V & $\mathrm{C}$ & 6 & 5754 & 4.71 & 0.32 & 0.3 \\
\hline HIP 29316 & 061054.80 & +101905.0 & 10.39 & 91.65 & $\mathrm{M} 2.5 \mathrm{~V}$ & V & 10 & 2567 & 52.81 & 1.27 & 15.4 \\
\hline HIP 30269 & 062202.50 & -052717.0 & 8.06 & 3.22 & F5V & V & 14 & 5389 & 20.29 & 14.85 & 99.0 \\
\hline HIP 33560 & 065826.05 & -125930.6 & 9.16 & 44.41 & K4V & V? & 14 & 8376 & -4.33 & 0.96 & 2.3 \\
\hline HIP 34341 & 070709.31 & +032650.7 & 9.87 & 38.26 & K5 & $\mathrm{S} 1$ & 21 & 5703 & -17.35 & $\ldots$ & $\ldots$ \\
\hline HIP 35706 & 072202.05 & +681627.6 & 10.10 & 23.84 & $\mathrm{~K} 5 \mathrm{~V}$ & $\mathrm{~S} 1$ & 21 & 5824 & -29.95 & $\ldots$ & $\ldots$ \\
\hline HIP 36758 & 073334.77 & +392714.0 & 9.75 & 5.95 & F8 & $\mathrm{V} ?$ & 11 & 1540 & 36.73 & 1.02 & 2.7 \\
\hline HIP 38195 & 074932.01 & +412808.3 & 9.41 & 8.98 & G5 & $\mathrm{C} ?$ & 10 & 6508 & 71.24 & 1.69 & 7.1 \\
\hline HIP 39681 & 080634.41 & +222724.2 & 7.22 & 14.73 & G5IV & $\mathrm{S} 1$ & 68 & 10984 & -0.57 & $\ldots$ & $\ldots$ \\
\hline HIP 40253 & $0813 \quad 17.31$ & +491315.5 & 8.54 & 8.59 & F5 & S1 & 67 & 6961 & -3.26 & $\ldots$ & $\ldots$ \\
\hline HIP 40724 & 081844.42 & -151208.5 & 9.87 & 28.85 & $\mathrm{~K} 5 \mathrm{~V}$ & $\mathrm{~V}$ & 2 & 797 & 88.15 & 1.05 & 7.6 \\
\hline HD 71028 & 082607.19 & +282410.7 & 8.01 & 2.21 & K0III & $\mathrm{S} 1$ & 29 & 7645 & 34.15 & $\ldots$ & $\ldots$ \\
\hline HIP 42507 & 084000.27 & -062833.1 & 9.88 & 38.80 & K6V & $\mathrm{C} ?$ & 5 & 2245 & -12.14 & 1.38 & 17.1 \\
\hline HIP 42550 & 084022.54 & +514506.6 & 7.70 & 1.70 & G5III & $\mathrm{C}$ & 11 & 10684 & -101.16 & 0.44 & 1.3 \\
\hline HD 73394B & 084018.25 & +514546.8 & 11.54 & 1.41 & - & s2 & 5 & 4133 & -32.54 & $\ldots$ & $\ldots$ \\
\hline HIP 43820 & 085524.82 & +704739.2 & 8.61 & 86.32 & M1V & $\mathrm{V}$ & 19 & 5226 & 44.64 & $\ldots$ & $\ldots$ \\
\hline HD 75632B & 085524.82 & +704739.2 & 8.86 & 86.32 & M1V & $\mathrm{C}$ & 2 & 326 & 43.81 & 0.08 & 0.3 \\
\hline HIP 46383 & 092728.37 & +393017.9 & 9.85 & 30.60 & $\mathrm{~K} 4 \mathrm{~V}$ & S2 & 12 & 2585 & -32.36 & $\ldots$ & $\ldots$ \\
\hline BD-08 2689 & 092851.52 & -091600.8 & 10.54 & 9.20 & MOV & $\mathrm{V}$ & 4 & 2208 & -14.81 & 1.55 & 24.5 \\
\hline HIP 46926 & 093352.46 & +152931.2 & 9.47 & 9.32 & G0 & S2 & 38 & 5046 & -10.86 & $\ldots$ & $\ldots$ \\
\hline
\end{tabular}


Table A.1. continued.

\begin{tabular}{|c|c|c|c|c|c|c|c|c|c|c|c|}
\hline Name & \multicolumn{2}{|c|}{ J2000 } & $\begin{array}{c}V \\
(\mathrm{mag})\end{array}$ & $\begin{array}{c}\varpi \\
(\mathrm{mas})\end{array}$ & $\begin{array}{l}\text { Sp. } \\
\text { type }\end{array}$ & Type & $N$ & $\begin{array}{l}\Delta T \\
\text { (d) }\end{array}$ & $\begin{array}{c}\langle\mathrm{RV}\rangle \\
\left(\mathrm{km} \mathrm{s}^{-1}\right)\end{array}$ & $\begin{array}{c}\mathrm{rms} \\
\left(\mathrm{km} \mathrm{s}^{-1}\right)\end{array}$ & $\chi^{2} /(N-1)$ \\
\hline HIP 47133 & 093615.91 & +373145.5 & 11.02 & 25.78 & MOV & $\mathrm{S} 2$ & 16 & 5819 & -0.68 & $\ldots$ & \\
\hline HIP 47899 & 094544.26 & +501408.3 & 11.19 & 12.58 & $\mathrm{~K} 4 \mathrm{~V}$ & S2 & 30 & 6588 & -15.01 & $\ldots$ & \\
\hline HIP 48346 & 095118.98 & +373615.9 & 9.96 & 19.28 & K8 & S2 & 23 & 2828 & -14.65 & $\ldots$ & . \\
\hline HIP 50156 & 101419.18 & +210429.6 & 10.08 & 42.74 & $\mathrm{M} 0.7 \mathrm{~V}$ & S1 & 13 & 2527 & 8.74 & $\ldots$ & 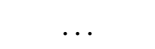 \\
\hline HIP 50271 & 101552.67 & +255001.2 & 9.44 & 5.77 & G0 & S1 & 49 & 10619 & 4.29 & $\ldots$ & \\
\hline HIP 52021 & 103747.41 & -062322.5 & 9.94 & 26.41 & $\mathrm{~K} 8$ & $\mathrm{~V}$ & 8 & 6592 & -11.58 & 7.11 & 99.0 \\
\hline HIP 54002 & 110250.12 & -09 1949.3 & 9.04 & 32.07 & $\mathrm{~K} 3 \mathrm{~V}$ & $\mathrm{~V}$ & 6 & 6554 & -6.78 & 0.98 & 9.4 \\
\hline HIP 54094 & 110407.16 & +532255.5 & 9.98 & 21.45 & $?$ & $\mathrm{~V} ?$ & 9 & 6554 & -23.88 & 0.58 & 4.2 \\
\hline HIP 56229 & 113136.39 & +403001.2 & 9.75 & 22.85 & M0 & $\mathrm{S} 2$ & 8 & 473 & 12.02 & $\ldots$ & $\ldots$ \\
\hline HIP 57058 & 114149.59 & +050826.5 & 9.59 & 32.26 & $\mathrm{~K} 4 \mathrm{~V}$ & S1 & 11 & 2602 & 18.90 & $\ldots$ & $\ldots$ \\
\hline $\mathrm{BD}+442120 \mathrm{~A}$ & 114348.16 & +441046.5 & 11.22 & 2.86 & F5 & $\mathrm{C}$ & 5 & 4136 & -5.73 & 0.74 & 3.6 \\
\hline $\mathrm{BD}+442120 \mathrm{~B}$ & 114347.90 & +441040.3 & 11.45 & 3.06 & $?$ & S1 & 17 & 4136 & -4.39 & $\ldots$ & \\
\hline $\mathrm{BD}+442120 \mathrm{C}$ & 114344.06 & +441049.1 & 11.04 & 3.06 & $?$ & $\mathrm{C}$ & 11 & 7654 & 15.01 & 0.57 & 0.6 \\
\hline GQ Leo & 114745.73 & +125403.4 & 10.83 & 16.29 & $\mathrm{~K} 5 \mathrm{Ve}$ & $\mathrm{C}$ & 12 & 5468 & -12.77 & 1.31 & 15.0 \\
\hline HIP 57857 & 115156.21 & +3307 11.4 & 10.97 & 18.50 & K0V & V & 11 & 6645 & -20.28 & 2.96 & 95.6 \\
\hline HIP 57949 & 115305.24 & +185548.1 & 11.73 & 32.00 & $\mathrm{M} 0.5 \mathrm{~V}$ & V & 7 & 5529 & 6.82 & 2.48 & 33.2 \\
\hline HIP 59000 & 120550.66 & -185230.9 & 10.02 & 42.72 & K5V & - & 1 & 0 & -8.20 & $\ldots$ & $\ldots$ \\
\hline HIP 60433 & 122326.85 & +201727.0 & 10.02 & 24.99 & K4V & $\mathrm{V}$ & 15 & 6594 & -40.01 & 6.20 & 99.0 \\
\hline HIP 60448 & 122334.71 & +275447.6 & 11.41 & 33.82 & $\mathrm{~K} 5 \mathrm{~V}$ & V & 5 & 6592 & -31.14 & 1.39 & 22.8 \\
\hline HIP 61436 & 123519.72 & +340406.6 & 10.51 & 24.43 & $\mathrm{~K} 5 \mathrm{~V}$ & $\mathrm{~S} 2$ & 24 & 2671 & -0.01 & & \\
\hline HIP 62505 & 124832.31 & -154310.1 & 7.89 & 39.21 & $\mathrm{~K} 2.5 \mathrm{~V}$ & $\mathrm{~V}$ & 12 & 4417 & -1.87 & 3.35 & 32.9 \\
\hline HIP 62755 & 125134.58 & +595025.9 & 11.31 & 0.81 & $\mathrm{~K}$ ? & S1 & 11 & 3000 & -47.03 & $\ldots$ & \\
\hline HIP 63253 & 125740.21 & +351330.1 & 10.68 & 46.84 & MOV & $\mathrm{V}$ & 11 & 2615 & -10.00 & 1.32 & 25.7 \\
\hline HIP 63816 & 130446.60 & +555410.1 & 10.75 & 30.63 & MoV & $\mathrm{C}$ & 3 & 2598 & -22.47 & 0.32 & 1.6 \\
\hline HIP 63942 & 130615.40 & +204345.3 & 9.40 & 53.18 & K4V & $\mathrm{C}$ & 13 & 5545 & -2.84 & 0.57 & 1.3 \\
\hline HIP 65012 & 131934.69 & +350624.5 & 11.90 & 73.99 & M3V & - & 1 & 0 & -5.20 & $\ldots$ & $\ldots$ \\
\hline HIP 65026 & 131945.65 & +474641.0 & 8.76 & 109.98 & $\mathrm{M} 2 \mathrm{~V}$ & S2 & 27 & 2629 & 0.12 & $\ldots$ & $\ldots$ \\
\hline HIP 65327 & 132323.30 & +575422.1 & 9.56 & 41.47 & $\mathrm{~K} 5 \mathrm{~V}$ & S1 & 15 & 6115 & -8.53 & $\ldots$ & $\ldots$ \\
\hline GJ 513 & 132921.31 & +112626.5 & 11.92 & 52.30 & M3V & $\mathrm{V}$ & 4 & 416 & 29.55 & 2.73 & 8.5 \\
\hline HIP 65887 & 133022.60 & +072454.5 & 7.63 & 3.29 & K0 & S1 & 25 & 3674 & -3.42 & $\ldots$ & \\
\hline HIP 66290 & 133511.42 & +222959.0 & 6.99 & 26.29 & F5V & S1 & 36 & 8395 & -14.04 & $\ldots$ & $\ldots$ \\
\hline HIP 67086 & 134502.39 & +020531.5 & 10.78 & 21.67 & K5 & $\mathrm{C}$ ? & 14 & 2886 & -27.23 & $\ldots$ & $\ldots$ \\
\hline BD+26 2498 & 134918.69 & +255254.2 & 9.85 & 1.40 & G5 & $\mathrm{S} 1$ & 31 & 6654 & -13.64 & $\ldots$ & $\ldots$ \\
\hline HIP 67808 & 135327.56 & +125633.4 & 9.77 & 45.62 & K7V & $\mathrm{V} ?$ & 6 & 2659 & -18.71 & 0.43 & 2.2 \\
\hline BD+19 2735 & 135813.62 & +191711.8 & 9.31 & 27.17 & $\mathrm{~K} 2$ & S1 & 20 & 5133 & -9.53 & $\ldots$ & $\ldots$ \\
\hline HIP 68801 & 140503.72 & +100048.9 & 8.68 & 19.58 & G5 & S2 & 24 & 5429 & -36.32 & $\ldots$ & $\ldots$ \\
\hline HIP 69549 & 141412.16 & +180506.8 & 7.98 & 11.72 & $\mathrm{G}$ & S2 & 29 & 6951 & 8.58 & $\ldots$ & $\ldots$ \\
\hline HIP 71904 & 144226.26 & +193012.7 & 10.03 & 42.24 & $\mathrm{~K} 5 \mathrm{~V}$ & $\mathrm{C}$ & 2 & 2550 & -28.08 & 0.09 & 0.2 \\
\hline HIP 71914 & 144233.81 & +192848.6 & 9.12 & 41.70 & $\mathrm{~K} 5 \mathrm{~V}$ & - & 1 & 0 & -28.70 & $\ldots$ & $\ldots$ \\
\hline HIP 72508 & 144932.38 & +512228.2 & 6.48 & 19.09 & F5IV & s2 & 11 & 7052 & -6.13 & $\ldots$ & $\ldots$ \\
\hline BD+49 2364 & 151325.00 & +490024.0 & 10.77 & 0.97 & $?$ & $\mathrm{~V}$ & 22 & 10789 & -74.33 & 1.69 & 19.7 \\
\hline HIP 76941 & 154238.57 & +315645.5 & 10.88 & 20.10 & $\mathrm{~K} 5 \mathrm{~V}$ & $\mathrm{~S} 1$ & 26 & 2886 & -12.50 & $\ldots$ & $\ldots$ \\
\hline HIP 77141 & 154500.29 & +355740.9 & 10.11 & 18.50 & $\mathrm{~K} 4 / 5 \mathrm{~V}$ & $\mathrm{~S} 1$ & 30 & 2913 & -3.46 & $\ldots$ & \\
\hline HIP 78158 & 155733.88 & +343222.8 & 10.83 & 19.30 & K5V & S1 & 19 & 2748 & -44.84 & $\ldots$ & $\ldots$ \\
\hline HIP 79796 & 161705.39 & +551609.1 & 9.46 & 49.35 & $\mathrm{M} 1.5 \mathrm{~V}$ & S1 & 14 & 2895 & -30.82 & 12.49 & 99.0 \\
\hline HIP 80751 & 162914.36 & +234633.9 & 10.08 & 30.84 & $\mathrm{~K} 5 \mathrm{~V}$ & $\mathrm{~S} 1$ & 14 & 6554 & -32.81 & $\ldots$ & $\ldots$ \\
\hline $\mathrm{BD}+521986 \mathrm{~A}$ & 163913.76 & +523739.4 & 10.01 & 22.86 & K8 & V & 13 & 2926 & -8.55 & 3.30 & 99.0 \\
\hline $\mathrm{BD}+521986 \mathrm{~B}$ & 163913.10 & +523738.2 & 11.40 & 22.71 & - & $\mathrm{C}$ & 2 & 0 & -8.69 & 0.39 & 6.9 \\
\hline HIP 82506 & 165146.46 & +252400.7 & 7.09 & 14.84 & F4III & $\mathrm{V}$ & 8 & 4785 & -25.39 & 4.76 & 99.0 \\
\hline $\mathrm{BD}+611678 \mathrm{C}$ & 173534.49 & +614053.6 & 9.97 & 69.83 & M1V & $\mathrm{C}$ & 9 & 3105 & -15.33 & 0.75 & 2.1 \\
\hline HIP 86221 & 173710.77 & +275347.2 & 9.20 & 32.00 & M0V & $\mathrm{C}$ & 2 & 2825 & -42.55 & 0.33 & 3.3 \\
\hline $\mathrm{BD}+272853 \mathrm{C}$ & 173711.41 & +275351.4 & 11.61 & 31.09 & K5 & - & 1 & 0 & -43.39 & $\ldots$ & $\ldots$ \\
\hline HIP 90274 & 182510.11 & +64 5018.3 & 6.86 & 5.70 & K0 & s2 & 28 & 5881 & -52.07 & $\ldots$ & \\
\hline HIP 91043 & 183420.10 & +184124.2 & 7.45 & 28.27 & G2V & s2 & 39 & 4879 & -22.21 & $\ldots$ & $\ldots$ \\
\hline HIP 92952 & 185615.93 & +543148.1 & 10.37 & 21.51 & MOV & S1 & 27 & 2944 & -18.38 & $\ldots$ & \\
\hline HIP 94557 & 191439.16 & +191903.7 & 11.54 & 55.24 & $\mathrm{M} 4.5 \mathrm{~V}$ & $\mathrm{~V}$ & 3 & 2904 & -4.28 & 19.34 & 99.0 \\
\hline
\end{tabular}


Table A.1. continued.

\begin{tabular}{|c|c|c|c|c|c|c|c|c|c|c|c|}
\hline Name & \multicolumn{2}{|c|}{$\begin{array}{ll}\mathrm{RA} & \mathrm{J} 2000 \\
\end{array}$} & $\begin{array}{c}V \\
(\mathrm{mag})\end{array}$ & $\begin{array}{c}\varpi \\
(\mathrm{mas})\end{array}$ & $\begin{array}{l}\text { Sp. } \\
\text { type }\end{array}$ & Type & $N$ & $\begin{array}{l}\Delta T \\
\text { (d) }\end{array}$ & $\begin{array}{c}\langle\mathrm{RV}\rangle \\
\left(\mathrm{km} \mathrm{s}^{-1}\right)\end{array}$ & $\begin{array}{c}\mathrm{rms} \\
\left(\mathrm{km} \mathrm{s}^{-1}\right)\end{array}$ & $\chi^{2} /(N-1)$ \\
\hline HIP 94622 & 191518.84 & +245349.5 & 9.78 & 34.57 & M0 & s2 & 7 & 2989 & -72.83 & . & $\cdots$ \\
\hline $\mathrm{BD}+77767$ & 201100.50 & +774318.4 & 10.30 & 24.54 & K8 & S1 & 18 & 2822 & -25.83 & & \\
\hline HIP 99969 & 201655.43 & +065518.3 & 9.47 & 22.67 & K4V & s2 & 8 & 2611 & -56.38 & .. & \\
\hline HIP 101941 & 203929.75 & +280518.6 & 7.78 & 2.63 & K4III & S1 & 46 & 4775 & -20.56 & .. & $\ldots$ \\
\hline HIP 102300 & 204341.37 & +641654.1 & 11.38 & 46.53 & MoVe & $\mathrm{V}$ & 5 & 2963 & 15.51 & 1.25 & 11.8 \\
\hline HIP 102320 & 204353.36 & +311910.4 & 9.96 & 24.06 & K5 & S2 & 29 & 3013 & -21.08 & & $\ldots$ \\
\hline HIP 102718 & 204850.72 & +051158.8 & 9.69 & 9.70 & F7Vw & $\mathrm{C}$ ? & 6 & 4290 & -117.67 & 0.64 & 2.8 \\
\hline HIP 103375 & 205637.80 & +524936.9 & 9.66 & 7.55 & G0 & $\mathrm{C} ?$ & 11 & 4418 & -47.55 & 0.53 & 2.5 \\
\hline HIP 104994 & 211554.95 & +285747.4 & 10.41 & 6.97 & G5 & S3 & 30 & 6669 & -46.87 & & \\
\hline HIP 105504 & 212207.78 & -103047.9 & 10.33 & 24.00 & K7 & $\mathrm{V}$ & 5 & 2613 & 7.40 & 17.45 & 99.0 \\
\hline $\mathrm{BD}+473439$ & 213048.00 & +482725.4 & 8.66 & 7.12 & K0 & s2 & 14 & 5465 & 10.04 & & \\
\hline HIP 110291 & 222023.85 & +462505.7 & 8.51 & 16.12 & G0 & S1 & 40 & 1571 & -108.04 & $\ldots$ & \\
\hline HIP 110526 & 222329.10 & +322733.9 & 10.76 & 64.47 & M3.0V & $\mathrm{C}$ & 13 & 2940 & -19.90 & 0.52 & 2.4 \\
\hline HIP 110978 & 222904.21 & -134201.8 & 9.17 & 1.25 & K2III & $\mathrm{V} ?$ & 3 & 34 & -1.37 & 0.82 & 6.3 \\
\hline HIP 111685 & 223729.90 & +392251.6 & 9.41 & 41.99 & MoVe & $\mathrm{S} 1$ & 10 & 5457 & -58.38 & $\ldots$ & \\
\hline HIP 111942 & 224030.30 & +430047.4 & 9.83 & 31.92 & K8V & S1 & 20 & 2940 & -31.76 & .. & $\ldots$ \\
\hline HIP 112040 & 224134.99 & +184927.5 & 10.51 & 31.85 & M0V & $\mathrm{V}$ & 13 & 5469 & -15.94 & 1.17 & 10.9 \\
\hline HIP 112268 & 224424.92 & +173323.8 & 10.19 & 19.83 & K6V & V & 13 & 5521 & -38.11 & 0.95 & 8.7 \\
\hline HIP 112523 & 224730.45 & +191327.4 & 10.29 & 26.95 & $\mathrm{~K} 5 \mathrm{~V}$ & S1 & 13 & 5458 & 3.97 & 1.86 & 28.7 \\
\hline HIP 116003 & 233013.44 & -202327.5 & 11.11 & 62.67 & $\mathrm{M} 2 \mathrm{Ve}$ & $\mathrm{V}$ & 2 & 7 & -6.83 & 7.07 & 91.2 \\
\hline BD+66 1664 & 235810.67 & +673359.7 & 8.73 & 11.13 & G5 & S1 & 33 & 1175 & -22.08 & & $\ldots$ \\
\hline HIP 118212 & 235843.51 & +464344.9 & 9.62 & 58.41 & K7V & S1 & 18 & 2986 & 1.00 & $\ldots$ & $\ldots$ \\
\hline
\end{tabular}


Table A.2. Spectroscopic orbits.

\begin{tabular}{|c|c|c|c|c|c|c|c|c|c|}
\hline Name & $\begin{array}{c}P \\
\text { (d) }\end{array}$ & $\begin{array}{c}T \\
(+2400000)\end{array}$ & $\begin{array}{c}e \\
(\mathrm{deg})\end{array}$ & $\begin{array}{c}\omega_{\mathrm{A}} \\
\left(\mathrm{km} \mathrm{s}^{-1}\right)\end{array}$ & $\begin{array}{c}K_{1} \\
\left(\mathrm{~km} \mathrm{~s}^{-1}\right)\end{array}$ & $\begin{array}{c}K_{2} \\
\left(\mathrm{~km} \mathrm{~s}^{-1}\right)\end{array}$ & $\begin{array}{c}\gamma \\
\left(\mathrm{km} \mathrm{s}^{-1}\right)\end{array}$ & $\mathrm{rms}_{1,2}$ & $\begin{array}{c}M_{1,2} \sin ^{3} i \\
\left(\mathcal{M}_{\odot}\right)\end{array}$ \\
\hline \multirow[t]{2}{*}{$\mathrm{BD}+334827 \mathrm{D}$} & 67.9618 & 51794.504 & 0.197 & 280.4 & 10.263 & $\cdots$ & -23.478 & 0.58 & $>0.2$ \\
\hline & \pm 0.0035 & \pm 0.633 & \pm 0.010 & \pm 3.6 & \pm 0.125 & & \pm 0.080 & & \\
\hline \multirow{2}{*}{ HIP 3428} & 97.250 & 58183.305 & 0.391 & 21.6 & 25.244 & 26.490 & -6.648 & 0.52 & 0.56 \\
\hline & \pm 0.015 & \pm 0.266 & \pm 0.005 & \pm 1.1 & \pm 0.197 & \pm 0.211 & \pm 0.072 & 0.46 & 0.53 \\
\hline \multirow[t]{2}{*}{ HD 8691} & 581.17 & 55965.5 & 0.544 & 313.5 & 2.366 & $\ldots$ & -36.183 & 0.54 & $>0.08$ \\
\hline & \pm 1.21 & \pm 5.1 & \pm 0.036 & \pm 5.5 & \pm 0.110 & $\ldots$ & \pm 0.065 & & $\ldots$ \\
\hline \multirow[t]{2}{*}{ HIP 9867} & 897.0 & 56004.0 & 0.0 & 0.0 & 8.057 & 11.347 & 61.419 & 0.38 & 0.40 \\
\hline & \pm 2.3 & \pm 5.8 & Fixed & Fixed & \pm 0.114 & \pm 0.184 & \pm 0.079 & 0.37 & 0.28 \\
\hline \multirow[t]{2}{*}{ HIP 10258} & 5.88554 & 54206.1875 & 0.0 & 0.0 & 66.011 & 64.231 & -59.148 & 0.92 & 0.66 \\
\hline & \pm 0.00001 & \pm 0.0022 & Fixed & Fixed & \pm 0.176 & \pm 0.189 & \pm 0.085 & 0.88 & 0.68 \\
\hline \multirow[t]{2}{*}{ HIP $13460 \mathrm{Aa}, \mathrm{Ab}$} & 76.433 & 58410.20 & 0.057 & 124.1 & 14.565 & 16.060 & $\ldots$ & 0.88 & 0.12 \\
\hline & \pm 0.014 & \pm 3.20 & \pm 0.016 & \pm 14.2 & \pm 0.254 & \pm 1.508 & & 3.23 & 0.11 \\
\hline \multirow[t]{2}{*}{ HIP $13460 \mathrm{AB}$} & 1862: & 55433 & 0.502 & 232.8 & 4.00 & $\ldots$ & -74.386 & $\ldots$ & $>0.29$ \\
\hline & \pm 25 & \pm 149 & \pm 0.186 & \pm 7.4 & \pm 2.04 & $\ldots$ & \pm 0.577 & & $\ldots$ \\
\hline \multirow[t]{2}{*}{ HIP 14478} & 1325.2 & 57011.8 & 0.369 & 142.4 & 5.814 & $\ldots$ & -50.871 & 0.22 & $>0.34$ \\
\hline & \pm 7.8 & \pm 16.5 & \pm 0.033 & \pm 5.8 & \pm 0.162 & $\ldots$ & \pm 0.183 & & $\ldots$ \\
\hline \multirow{2}{*}{ HIP 14864} & 59.428 & 56470.94 & 0.642 & 130.3 & 27.626 & 31.667 & -31.907 & 0.37 & 0.33 \\
\hline & \pm 0.005 & \pm 0.07 & \pm 0.006 & \pm 0.8 & \pm 0.256 & \pm 1.020 & \pm 0.138 & 1.15 & 0.29 \\
\hline \multirow[t]{2}{*}{ HIP 17102} & 183.113 & 57305.62 & 0.6115 & 350.09 & 25.134 & 24.121 & 4.273 & 0.42 & 0.56 \\
\hline & \pm 0.019 & \pm 0.17 & \pm 0.0020 & \pm 0.49 & \pm 0.124 & \pm 0.128 & \pm 0.073 & 0.41 & 0.58 \\
\hline \multirow[t]{2}{*}{ HIP 18448} & 1538.9 & 54793.6 & 0.478 & 210.6 & 6.469 & $\ldots$ & 16.657 & 0.34 & $>0.38$ \\
\hline & \pm 2.6 & \pm 5.9 & \pm 0.018 & \pm 2.3 & \pm 0.117 & $\ldots$ & \pm 0.088 & & \\
\hline \multirow[t]{2}{*}{ HIP 19915} & 7.30032 & 50087.8789 & 0.0 & 0.0 & 27.326 & 30.620 & -8.157 & 1.25 & 0.078 \\
\hline & \pm 0.00003 & \pm 0.0092 & Fixed & Fixed & \pm 0.160 & \pm 0.182 & \pm 0.107 & 1.44 & 0.069 \\
\hline \multirow[t]{2}{*}{ HD 279846} & 15.4860 & 55271.598 & 0.039 & 245.1 & 44.723 & 47.041 & 12.429 & 0.78 & 0.63 \\
\hline & \pm 0.0001 & \pm 0.189 & \pm 0.003 & \pm 4.5 & \pm 0.217 & \pm 0.225 & \pm 0.075 & 1.09 & 0.60 \\
\hline \multirow[t]{2}{*}{ HIP 20709} & 140.748 & 56222.61 & 0.557 & 280.0 & 23.200 & 31.750 & 44.060 & 0.19 & 0.84 \\
\hline & \pm 0.020 & \pm 0.34 & \pm 0.007 & \pm 0.6 & \pm 0.086 & \pm 0.332 & \pm 0.067 & 0.19 & 0.60 \\
\hline \multirow[t]{2}{*}{ HIP 21710} & 610.43 & 56635.1 & 0.415 & 269.9 & 4.596 & $\ldots$ & -27.124 & 0.75 & $>0.17$ \\
\hline & \pm 0.15 & \pm 4.2 & \pm 0.018 & \pm 3.4 & \pm 0.099 & & \pm 0.067 & & \\
\hline HIP 28663 & 8.5542 & 52158.14 & 0.079 & 255.3 & 30.129 & $\ldots$ & -25.105 & 0.68 & $>0.35$ \\
\hline & \pm 0.0001 & \pm 0.12 & \pm 0.006 & \pm 5.0 & \pm 0.229 & $\ldots$ & \pm 0.136 & & $\ldots$ \\
\hline HIP 34341 & 875.37 & 55875.7 & 0.507 & 328.7 & 4.561 & $\ldots$ & -17.353 & 0.45 & $>0.20$ \\
\hline & \pm 1.69 & \pm 8.6 & \pm 0.026 & \pm 5.4 & \pm 0.284 & & \pm 0.106 & $\ldots$ & $\ldots$ \\
\hline HIP 35706 & 4741 & 56641 & 0.337 & 266.5 & 5.639 & $\ldots$ & -29.953 & 0.22 & $>0.57$ \\
\hline & \pm 73 & \pm 41 & \pm 0.022 & \pm 4.6 & \pm 0.098 & $\ldots$ & \pm 0.125 & & $\ldots$ \\
\hline HIP 39681 & 1717.7 & 51745.8 & 0.508 & 76.3 & 8.077 & $\ldots$ & -0.569 & 0.47 & $>0.51$ \\
\hline & \pm 0.7 & \pm 3.7 & \pm 0.009 & \pm 1.5 & \pm 0.116 & & \pm 0.054 & $\ldots$ & $\ldots$ \\
\hline HIP 40253 & 897.83 & 54881 & 0.035 & 340 & 6.638 & $\ldots$ & -3.255 & 0.51 & $>0.37$ \\
\hline & \pm 0.86 & \pm 66 & \pm 0.010 & \pm 26.5 & \pm 0.151 & & \pm 0.060 & & $\ldots$ \\
\hline HD 71028 & 1129.5 & 53846.9 & 0.147 & 166.1 & 4.989 & $\ldots$ & 34.147 & 0.41 & $>0.29$ \\
\hline & \pm 3.2 & \pm 42.6 & \pm 0.028 & \pm 14.2 & \pm 0.174 & & \pm 0.086 & $\ldots$ & $\ldots$ \\
\hline HIP 46383 & 8.490802 & 57396.839 & 0.0987 & 324.3 & 56.286 & 57.002 & -32.322 & 0.38 & 0.63 \\
\hline & \pm 0.000003 & \pm 0.013 & \pm 0.0016 & \pm 0.6 & \pm 0.095 & \pm 0.096 & \pm 0.005 & 0.43 & 0.63 \\
\hline HIP 46926 & 3.10662 & 54604.7305 & 0.0 & 0.0 & 22.600 & 26.464 & -10.859 & 0.59 & 0.020 \\
\hline & \pm 0.00001 & \pm 0.0025 & Fixed & Fixed & \pm 0.127 & \pm 0.185 & \pm 0.065 & 0.50 & 0.017 \\
\hline HIP 47133 & 4.38804 & 58055.344 & 0.474 & 140.2 & 69.252 & 74.379 & -0.677 & 2.71 & 0.47 \\
\hline & \pm 0.00001 & \pm 0.015 & \pm 0.005 & \pm 1.9 & \pm 2.035 & \pm 2.506 & \pm 0.257 & 2.79 & 0.44 \\
\hline HIP 47899 & 146.309 & 56614.18 & 0.075 & 5.8 & 20.100 & 21.360 & -15.010 & 0.66 & 0.55 \\
\hline & \pm 0.020 & \pm 1.50 & \pm 0.005 & \pm 3.6 & \pm 0.131 & \pm 0.144 & \pm 0.071 & 0.63 & 0.52 \\
\hline HIP 48346 & 79.013 & 56464.52 & 0.695 & 187.9 & 26.408 & 36.202 & -14.648 & 0.86 & 0.43 \\
\hline & \pm 0.073 & \pm 1.03 & \pm 0.041 & \pm 2.5 & \pm 3.209 & \pm 4.602 & \pm 0.308 & 2.82 & 0.31 \\
\hline HIP 50156 & 73.225 & 56288.11 & 0.140 & 308.7 & 9.851 & - & 8.741 & 0.63 & $>0.22$ \\
\hline & \pm 0.030 & \pm 2.10 & \pm 0.027 & \pm 11.1 & \pm 0.266 & & \pm 0.222 & & \\
\hline HIP 50271 & 47.4632 & 49595.278 & 0.688 & 55.6 & 28.703 & $\ldots$ & 4.288 & 0.98 & $>0.45$ \\
\hline & \pm 0.0003 & \pm 0.028 & \pm 0.003 & \pm 0.6 & \pm 0.156 & & \pm 0.108 & $\ldots$ & $\ldots$ \\
\hline HIP 56229 & 186.3: & 58096.59 & 0.549 & 345.7 & 22.183 & 23.033 & 12.015 & 0.42 & 0.53 \\
\hline & \pm 0.73 & \pm 1.04 & \pm 0.050 & \pm 1.7 & \pm 2.298 & \pm 2.386 & \pm 0.064 & 0.33 & 0.51 \\
\hline
\end{tabular}


Table A.2. continued.

\begin{tabular}{|c|c|c|c|c|c|c|c|c|c|}
\hline Name & $\begin{array}{c}P \\
(\mathrm{~d})\end{array}$ & $\begin{array}{c}T \\
(+2400000) \\
\end{array}$ & $\begin{array}{c}e \\
(\mathrm{deg})\end{array}$ & $\begin{array}{c}\omega_{\mathrm{A}} \\
\left(\mathrm{km} \mathrm{s}^{-1}\right)\end{array}$ & $\begin{array}{c}K_{1} \\
\left(\mathrm{~km} \mathrm{~s}^{-1}\right)\end{array}$ & $\begin{array}{c}K_{2} \\
\left(\mathrm{~km} \mathrm{~s}^{-1}\right)\end{array}$ & $\begin{array}{c}\gamma \\
\left(\mathrm{km} \mathrm{s}^{-1}\right)\end{array}$ & $\mathrm{rms}_{1,2}$ & $\begin{array}{c}M_{1,2} \sin ^{3} i \\
\left(\mathcal{M}_{\odot}\right)\end{array}$ \\
\hline \multirow[t]{2}{*}{ HIP 57058} & 725.9 & 57934.3 & 0.164 & 168.0 & 8.065 & $\cdots$ & 19.708 & 0.53 & $>0.50$ \\
\hline & \pm 0.9 & \pm 61.7 & \pm 0.079 & \pm 28.9 & \pm 1.128 & & \pm 0.603 & & \\
\hline \multirow[t]{2}{*}{$\mathrm{BD}+442120 \mathrm{~B}$} & 2.22596 & 55870.6875 & 0.0 & 0.0 & 46.215 & $\ldots$ & -4.387 & 1.15 & $>0.35$ \\
\hline & \pm 0.00001 & \pm 0.0038 & Fixed & Fixed & \pm 0.322 & $\ldots$ & \pm 0.218 & & \\
\hline \multirow{2}{*}{ HIP 61436} & 299.55 & 56819.02 & 0.457 & 260.7 & 17.590 & 19.677 & -0.363 & 0.48 & 0.49 \\
\hline & \pm 0.18 & \pm 1.24 & \pm 0.013 & \pm 1.9 & \pm 0.294 & \pm 0.491 & \pm 0.135 & 0.96 & 0.46 \\
\hline \multirow[t]{2}{*}{ HIP 62755} & 2238 & 56347.9 & 0.140 & 27.3 & 8.027 & $\ldots$ & -47.032 & 0.11 & $>0.69$ \\
\hline & \pm 27 & \pm 127.6 & \pm 0.024 & \pm 20.9 & \pm 0.419 & $\ldots$ & \pm 0.167 & & \\
\hline \multirow[t]{2}{*}{ HIP 65026} & 446.87 & 57009.7 & 0.082 & 12.2 & 9.129 & $\ldots$ & 0.109 & 0.65 & $0.65:$ \\
\hline & \pm 0.43 & \pm 10.1 & \pm 0.014 & \pm 8.4 & \pm 0.158 & $\ldots$ & \pm 0.104 & & $>0.29$ \\
\hline \multirow[t]{2}{*}{ HIP 65327} & 4354.0 & 56827.0 & 0.497 & 115.2 & 5.030 & $\ldots$ & -8.528 & 0.21 & $>0.45$ \\
\hline & \pm 13.8 & \pm 55.8 & \pm 0.051 & \pm 8.7 & \pm 0.557 & $\ldots$ & \pm 0.20 & & \\
\hline \multirow[t]{2}{*}{ HIP 65887} & 1200.0 & 53733.7 & 0.078 & 68.6 & 6.143 & $\ldots$ & -3.416 & 0.45 & $>0.38$ \\
\hline & \pm 3.0 & \pm 47.8 & \pm 0.020 & \pm 14.5 & \pm 0.134 & $\ldots$ & \pm 0.074 & $\ldots$ & \\
\hline \multirow[t]{2}{*}{ HIP 66290} & 1967 & 51294.7 & 0.681 & 78.5 & 6.731 & $\ldots$ & -14.044 & 0.54 & $>0.36$ \\
\hline & \pm 11 & \pm 9.1 & \pm 0.018 & \pm 5.8 & \pm 0.315 & $\ldots$ & \pm 0.139 & & \\
\hline \multirow[t]{2}{*}{ HIP 67086} & 40.871 & 56774.02 & 0.318 & 208.9 & 28.116 & 33.602 & -27.226 & 0.55 & 0.46 \\
\hline & \pm 0.007 & \pm 0.34 & \pm 0.024 & \pm 2.8 & \pm 0.564 & \pm 1.070 & \pm 0.255 & 0.54 & 0.39 \\
\hline \multirow[t]{2}{*}{ BD+26 2498} & 1536.2 & 53208.1 & 0.190 & 258.3 & 12.063 & $\ldots$ & -13.641 & 0.52 & $>1.02$ \\
\hline & \pm 1.8 & \pm 15.0 & \pm 0.010 & \pm 3.5 & \pm 0.117 & $\ldots$ & \pm 0.100 & & \\
\hline \multirow[t]{2}{*}{ BD+19 2735} & 3616 & 55769.8 & 0.607 & 89.8 & 4.913 & $\ldots$ & -9.535 & 0.28 & $>0.34$ \\
\hline & \pm 69 & \pm 33.2 & \pm 0.176 & \pm 7.9 & \pm 1.566 & $\ldots$ & \pm 0.120 & $\ldots$ & \\
\hline \multirow{2}{*}{ HIP 68801} & 2.84019 & 54536.9453 & 0.0 & 0.0 & 86.676 & 88.273 & -36.319 & 1.10 & 0.79 \\
\hline & \pm 0.00001 & \pm 0.0009 & Fixed & Fixed & \pm 0.173 & \pm 0.222 & \pm 0.099 & 0.90 & 0.78 \\
\hline \multirow[t]{2}{*}{ HIP 69549} & 6.03150 & 54087.0820 & 0.0 & 0.0 & 64.754 & 65.831 & 8.578 & 1.13 & 0.70 \\
\hline & \pm 0.00001 & \pm 0.0016 & Fixed & Fixed & \pm 0.132 & \pm 0.139 & \pm 0.068 & 1.36 & 0.69 \\
\hline \multirow[t]{2}{*}{ HIP 76941} & 267.06 & 57157.05 & 0.492 & 315.3 & 7.054 & $\ldots$ & -12.497 & 0.38 & $>0.23$ \\
\hline & \pm 0.31 & \pm 1.36 & \pm 0.012 & \pm 1.7 & \pm 0.122 & $\ldots$ & \pm 0.071 & & \\
\hline \multirow{2}{*}{ HIP 77141} & 17.3120 & 58299.184 & 0.836 & 14.2 & 50.441 & $\ldots$ & -3.460 & 0.28 & $>0.34$ \\
\hline & \pm 0.0005 & \pm 0.070 & \pm 0.154 & \pm 8.1 & \pm 58.975 & $\ldots$ & \pm 2.167 & $\ldots$ & . \\
\hline \multirow[t]{2}{*}{ HIP 78158} & 322.04 & 57083.48 & 0.395 & 299.7 & 11.784 & $\ldots$ & -44.839 & 0.34 & $>0.44$ \\
\hline & \pm 0.16 & \pm 1.43 & \pm 0.014 & \pm 1.9 & \pm 0.219 & $\ldots$ & \pm 0.099 & $\ldots$ & $\ldots$ \\
\hline HIP 80751 & 5252 & 58096.9 & 0.188 & 64.5 & 3.031 & $\ldots$ & -32.748 & 0.23 & $>1.02$ \\
\hline & \pm 324 & \pm 692.7 & \pm 0.069 & \pm 55.1 & \pm 0.748 & $\ldots$ & \pm 0.582 & & \\
\hline HIP 92952 & 7.9461 & 57609.65 & 0.044 & 291.9 & 17.290 & $\ldots$ & -18.380 & 0.40 & $>0.18$ \\
\hline & \pm 0.0002 & \pm 0.28 & \pm 0.007 & \pm 12.8 & \pm 0.148 & $\ldots$ & \pm 0.133 & $\ldots$ & $\ldots$ \\
\hline BD+77 767 & 17.3231 & 56737.1289 & 0.391 & 225.5 & 40.434 & $\ldots$ & -25.831 & 0.32 & $>0.63$ \\
\hline & \pm 0.0002 & \pm 0.0329 & \pm 0.005 & \pm 0.9 & \pm 0.331 & $\ldots$ & \pm 0.114 & & $\ldots$ \\
\hline HIP 101941 & 1026.69 & 54442.6 & 0.223 & 211.8 & 9.975 & $\ldots$ & -20.562 & 0.43 & $>0.64$ \\
\hline & \pm 1.37 & \pm 7.8 & \pm 0.012 & \pm 2.8 & \pm 0.111 & $\ldots$ & \pm 0.075 & $\ldots$ & \\
\hline HIP 102320 & 21.1854 & 57143.617 & 0.416 & 322.1 & 40.879 & 41.954 & -21.080 & 0.30 & 0.47 \\
\hline & \pm 0.0002 & \pm 0.016 & \pm 0.003 & \pm 0.3 & \pm 0.141 & \pm 0.206 & \pm 0.049 & 0.19 & 0.46 \\
\hline HIP 104994 & 51.82128 & 55970.823 & 0.407 & 303.6 & 25.796 & 32.988 & -46.869 & 0.85 & 0.48 \\
\hline & \pm 0.0015 & \pm 0.087 & \pm 0.003 & \pm 0.9 & \pm 0.090 & \pm 0.152 & \pm 0.062 & 0.49 & 0.37 \\
\hline HIP 110291 & 764.8 & 54423.0 & 0.563 & 3.9 & 3.888 & $\ldots$ & -108.035 & 0.52 & $>0.15$ \\
\hline & \pm 3.5 & \pm 4.7 & \pm 0.028 & \pm 4.0 & \pm 0.219 & $\ldots$ & \pm 0.088 & $\ldots$ & $\ldots$ \\
\hline HIP 111685 & 6177 & 54730 & 0.249 & 300.1 & 3.752 & $\ldots$ & -58.378 & 0.33 & $>0.40$ \\
\hline & \pm 26 & \pm 22 & \pm 0.007 & \pm 1.3 & \pm 0.154 & $\ldots$ & \pm 0.113 & $\ldots$ & \\
\hline HIP 111942 & 375.098 & 56849.88 & 0.437 & 339.5 & 7.880 & $\ldots$ & -32.470 & 0.32 & $>0.30$ \\
\hline & \pm 0.038 & \pm 1.39 & \pm 0.011 & \pm 0.4 & \pm 0.080 & $\ldots$ & \pm 0.070 & $\ldots$ & $\ldots$ \\
\hline HIP 112523 & 3255: & 55676.2 & 0.480 & 190.5 & 3.871 & $\ldots$ & 4.154 & 0.58 & $>0.36$ \\
\hline & \pm 50 & \pm 41.7 & Fixed & \pm 6.2 & \pm 0.243 & & \pm 0.156 & & \\
\hline BD+66 1664 & 3.09995 & 53785.0117 & 0.0 & 0.0 & 23.940 & $\ldots$ & -22.083 & 0.67 & $>0.18$ \\
\hline & \pm 0.00004 & \pm 0.0036 & Fixed & Fixed & \pm 0.174 & $\ldots$ & \pm 0.123 & $\ldots$ & $\ldots$ \\
\hline HIP 118212 & 872.90 & 56678.80 & 0.536 & 135.4 & 8.428 & $\ldots$ & 0.999 & 0.36 & $>0.40$ \\
\hline & \pm 1.23 & \pm 2.71 & \pm 0.016 & \pm 2.5 & \pm 0.138 & $\ldots$ & \pm 0.131 & $\ldots$ & $\ldots$ \\
\hline
\end{tabular}

